\title{
TRAVELING WAVE SOLUTIONS FOR BISTABLE DIFFERENTIAL-DIFFERENCE EQUATIONS WITH PERIODIC DIFFUSION*
}

\author{
CHRISTOPHER E. ELMER ${ }^{\dagger}$ AND ERIK S. VAN VLECK ${ }^{\ddagger}$
}

\begin{abstract}
We consider traveling wave solutions to spatially discrete reaction-diffusion equations with nonlocal variable diffusion and bistable nonlinearities. To find the traveling wave solutions we introduce an ansatz in which the wave speed depends on the underlying lattice as well as on time. For the case of spatially periodic diffusion we obtain analytic solutions for the traveling wave problem using a piecewise linear nonlinearity. The formula for the wave forms is implicitly defined in the general periodic case and we provide an explicit formula for the case of period two diffusion. We present numerical studies for time $t=0$ fixed and for the time evolution of the traveling waves. When $t=0$ we study the cases of homogeneous, period two, and period four diffusion coefficients using a cubic nonlinearity, and uncover, numerically, a period doubling bifurcation in the wave speed versus detuning parameter relation. For the time evolution case we also discover a detuning parameter dependent bifurcation in observed phenomena, which is a product of both the nonlocal diffusion operator and the spinodal effects of the nonlinearity.
\end{abstract}

Key words. traveling waves, propogation failure, bistable equation, periodic diffusion

AMS subject classifications. 35K57, 74N99

PII. S0036139999357113

1. Introduction. Problems that are modeled by nonlinear spatially discrete diffusion equations occur in many areas of science. In the materials sciences examples include crystal growth, interface motion in crystalline materials, spinodal decomposition, and grain boundary movement in thin films. Spatially discrete diffusion operators allow the material being modeled to be described in terms of its underlying crystalline lattice. Phenomena such as crystallographic pinning and lattice anisotropy, phenomena that are often added to spatially continuous models in an "ad hoc" manner, occur naturally in spatially discrete materials models. In biology, examples of applications of spatially discrete models include the bidomain model for cardiac tissue (defibrillation), tissue filtration, gas exchange in lungs, and calcium waves. Propagation failure and directional dependence are phenomena that occur in spatially discrete biological models that do not occur in their continuous counterparts. A relatively unexplored area of mathematics is spatially discrete diffusion equations with diffusion coefficients that depend on location, a necessary condition to effectively model many systems.

As a specific example, from [4], consider a material consisting of two phases and the variation in free energy that an interface between the phases encounters as it advances through a crystalline medium in a direction normal to the lattice planes, and let $v_{j}$ be a time dependent variable that indicates the phase of the $j$ th plane.

*Received by the editors October 4, 1999; accepted for publication (in revised form) September 20, 2000; published electronically February 8, 2001.

http://www.siam.org/journals/siap/61-5/35711.html

${ }^{\dagger}$ Material Science and Engineering Laboratory, National Institute of Science and Technology, Gaithersburg, MD 20899. Current address: Department of Mathematical Sciences, New Jersey Institute of Technology, Newark, NJ (elmer@m.njit.edu).

${ }_{\ddagger}$ Department of Mathematical and Computer Sciences, Colorado School of Mines, Golden, CO 80401 (evanvlec@mines.edu). The research of this author was supported by NIST contract 43NANB714674 and by NSF grants DMS-9505049 and DMS-9973393. 
Then the excess free energy $G$ per unit area of interface can be represented by a sum over the lattice planes of the form

$$
G=\sum_{j=-\infty}^{\infty}\left[F\left(v_{j}\right)+\frac{K}{\beta^{2}}\left(v_{j+1}-v_{j}\right)^{2}\right], \quad v_{-\infty}=0, \quad v_{+\infty}=1
$$

where $\beta$ is the interplaner distance, and $K$ is a possibly constant parameter which relates the effect of gradient to that of the increase in the free energy, $F\left(v_{j}\right)$, accompanying the creation of a unit volume of homogeneous material from the material reservoirs of the two bulk phases. Fixing $K$ constant might indicate that the temperature or perhaps the concentration are constant throughout the material. It is generally assumed that the interplaner distance $\beta$ is fixed. But interplaner distance, temperature, and concentration can all be spatially dependent. We wish to allow either $\beta \equiv \beta_{j}$ and/or $K=K_{j}$ to depend on spatial position. Knowledge of diffusion in such materials is necessary to describe the material's kinetic reactions.

Mathematical models of the behavior of axons often come from postulating an equivalent electrical circuit model (cable theory) of the excitable axonal membrane. As a second example, from [18], consider nerve fibers (axons) coated with a material called myelin (mylinated) with periodic gaps (nodes) in the coating. The action potential jumps from node to node along the fiber. Assuming the axial currents are constant, the intracellular, $I_{i}$, and extracellular, $I_{e}$, currents between nodes are

$$
I_{i}=-\frac{1}{r_{i}} \frac{V_{i, n+1}-V_{i, n}}{L}, \quad I_{e}=-\frac{1}{r_{e}} \frac{V_{e, n+1}-V_{e, n}}{L},
$$

where $L$ is the length of the myelin sheath between nodes, $r_{i}$ and $r_{e}$ are the intracellular and extracellular resistances per unit length of material, and $V_{i, n}$ and $V_{e, n}$ are the intracellular and extracellular voltages in the $n$th node. The total transmembrane current at a node $n$ is thus given by

$$
p\left(C \frac{\partial V_{n}}{\partial t}+I_{i o n}\right)=\frac{1}{\mu L\left(r_{i}+r_{e}\right)}\left(V_{n+1}-2 V_{n}+V_{n-1}\right),
$$

where $\mu$ is the length of a node, $p$ is the perimeter length of the axon, $C$ is the capacitance, and $I_{i o n}$ is the ionic current. Note that typically $p, \mu$, and $L$ are considered constant. We want to consider allowing any of these parameter to depend on space.

In this paper we consider traveling wave solutions to equations of the form

$$
\dot{u}_{j}(t)=L_{D} u_{j}(t)-f\left(u_{j}(t), a\right)
$$

spatially discrete reaction-diffusion equation with diffusion coefficients that vary on an integer lattice, where $u_{j}(t)$ maps $\mathbb{R}^{+} \cup\{0\} \rightarrow \mathbb{R}, j \in \mathbb{Z}$, indicates a particular element of the one-dimensional lattice, and " " denotes differentiation with respect to $t$. We represent variable diffusion over the spatially discrete domain with the operator $L_{D}$, a difference Laplacian operator of the form

$$
L_{D} u_{j}(t)=\alpha_{j+1}\left[u_{j+1}(t)-u_{j}(t)\right]+\alpha_{j}\left[u_{j-1}(t)-u_{j}(t)\right],
$$

where $\alpha_{j} \in \mathbb{R}^{+}, j \in \mathbb{Z}$. This is one choice of several equivalent divergence forms for the operator $L_{D}$. Our interest is in bistable systems, systems where $f(u, a)$ can be represented by a cubic-like nonlinear function with stable zeros at 0 and 1 , and an 
unstable zero at the detuning parameter $a \in(0,1)$. In the first half of this paper we construct analytical solutions using the piecewise linear nonlinearity

$$
f_{1}(u, a)=\left\{\begin{array}{cc}
u, & u<a \\
{[a-1, a],} & u=a \\
u-1, & u>a
\end{array}\right.
$$

and in the second half we consider numerical solutions using the cubic nonlinearity

$$
f_{2}(u, a)=d u(u-a)(u-1) .
$$

If we consider the change in $v_{j}$ in time per unit area of (1.1) and we allow $\beta$ and $K$ to vary with position, we obtain the spatially discrete reaction-diffusion equation

$$
\dot{v}_{j}=\gamma_{j+1}\left[v_{j+1}-v_{j}\right]+\gamma_{j}\left[v_{j-1}-v_{j}\right]-\frac{d F\left(v_{j}\right)}{d v_{j}},
$$

where $\gamma_{j}=2 K_{j} / \beta_{j}^{2}$. With the change of variables $\tau=t /(C R)$ and allowing $\mu, p$, and $L$ to vary along the axon, (1.2) becomes

$$
\frac{d V_{n}}{d \tau}=\rho_{j+1}\left[V_{j+1}-V_{j}\right]+\rho_{j}\left[V_{j-1}-V_{j}\right]-R I_{i o n},
$$

where $\rho_{j}=R /\left(\mu_{j} L_{j} p_{j}\left(r_{i}+r_{e}\right)\right)$. The nonlinearities (1.5) and (1.6) are both natural forms for the term $d F(u) / d u$ in (1.7) and for the term $R I_{i o n}$ in (1.8).

Our consideration of (1.3) with the spatially varying diffusion operator defined by (1.4) is motivated by the work of Fife [11] and Fife and Hsiao [12] who consider constant diffusion reaction-diffusion PDEs having a bistable nonlinearity $f$ with a spatial dependence and Shen [22] and [23] who considers both spatially discrete and spatially continuous constant diffusion reaction-diffusion equations where the bistable $f$ has an almost periodic time dependence. For spatially homogeneous diffusion, propagation failure, the failure of the wave to propagate for values of the detuning parameter $a$ over a nontrivial interval, in one-dimensional spatially discrete reaction-diffusion equations was studied by Keener [16], [17]. Zinner and his coworkers provided existence and stability of traveling wave solutions for spatially discrete reaction-diffusion equations [15], [25], [26], [27], [28]. Motivated by the works of Keener and Zinner, in [5] Cahn, Mallet-Paret, and Van Vleck provide results on propagation failure and lattice induced anisotropy for traveling wave solutions of two dimensional spatially discrete reaction-diffusion equations with nonlinearity (1.5). In [19] Mallet-Paret develops a Fredholm theory for equations with backward and forward delay terms and uses these results in [20] to investigate the global structure of traveling wave solutions of spatially discrete reaction-diffusion equations. We also mention here the related works on traveling wave solutions of spatially discrete reaction-diffusion type equations of Fath [10], Gao [14], and the seminal work of Weinberger [24].

In previous works traveling wave solutions to the constant diffusion coefficient equation

$$
\dot{u}(\eta, t)=L_{D} u(\eta, t)-f(u(\eta, t), a)
$$

with

$$
L_{D} u(\eta, t)=\sum_{k=1}^{n} \varepsilon_{k}[u(\eta+1, t)-2 u(\eta, t)+u(\eta-1, t)]
$$


are sometimes found by substituting the traveling wave ansatz $u(\eta, t)=\varphi(\eta \cdot \sigma-c t)$, where $c$ is the constant unknown wave speed and $\sigma$ is the direction normal. Our approach in this work is to allow the unknown wave speed to depend on the lattice and on time. We substitute the traveling wave ansatz

$$
u_{j}(t)=\phi\left(j-\int_{0}^{t} c_{j}(s) d s\right)
$$

with the unknown wave speed $c_{j}(t)$ into (1.3) to obtain the equations

$$
-c_{j}(t) \phi^{\prime}\left(\xi_{j}\right)=L_{T} \phi\left(\xi_{j}\right)-f\left(\phi\left(\xi_{j}\right), a\right),
$$

where

$$
L_{T} \phi\left(\xi_{j}\right)=\alpha_{j+1}\left[\phi\left(\xi_{j+1}\right)-\phi\left(\xi_{j}\right)\right]+\alpha_{j}\left[\phi\left(\xi_{j-1}\right)-\phi\left(\xi_{j}\right)\right],
$$

$\xi_{j}=j-\int_{0}^{t} c_{j}(s) d s$, and $\xi_{j \pm 1}=\xi_{j} \pm 1-\int_{0}^{t}\left[c_{j \pm 1}(s)-c_{j}(s)\right] d s$. One of the primary differences in solving (1.10) as opposed to solving constant diffusion traveling wave equations is that in (1.10) the forward and backward delays depend on the lattice and on time.

In section 2 we complete the definition of the problem, define boundary conditions, and choose a phase condition. This includes a change of variables based on $t$ being fixed and $\alpha_{j}$ being periodic in $j$. Next, using the Fourier integral transform, in section 3 we derive a solution to the periodic version of $(1.9)$ when $c_{j}(t)$ is not equal to zero for all $j \in \mathbb{Z}$ and $t \in \mathbb{R}^{+} \cup\{0\}$. Since the general periodic case is rather cumbersome algebraically, in section 4 , we demonstrate the periodic coefficient result for an equation of the form (1.9) with period $P=2$, along with its $a\left(c_{j}\right)$ relation. Section 5 is an introduction to the numerical results which includes necessary background. All of our numerical results are for our periodic diffusion differential-difference equation with the cubic nonlinearity, $f_{2}$. Our first series of examples, section 6 , is a study of periodic solution results for $t=0$ fixed. In this section we typically see period $P$ wave speeds when solving, depending on the location in the lattice. Included in this section is the constant diffusion coefficient problem with the spatially varying wave speed traveling wave ansatz. This problem generates an $a$ versus $c_{k}$ plot which contains bifurcation points dependent on the parameter $a$. The second numerical results section, section 7 , is a study of the time evolution of a period two problem. We present $u_{j}(t)$ solution plots along with a study of how the forward and backward delays in (1.10) evolve in time $t$. The types of behavior in the evolution of the delays exhibit a bifurcation which is also dependent on the detuning parameter $a$. We finish with a discussion and conclusions in section 8 .

2. Spatially periodic diffusion. The diffusion coefficient $\alpha_{j}$ describes the diffusion interaction between the lattice points at $j-1$ and at $j$ for all $j \in \mathbb{Z}$. Consider system (1.3) with diffusion coefficient $\alpha_{j}$ that varies periodically on $\mathbb{Z}$ with period $P=N_{l}+N_{r}+1, N_{l}, N_{r} \in \mathbb{Z}^{+} \cup\{0\}$, i.e.,

$$
\alpha_{j}=\alpha_{j+P}, \quad j \in \mathbb{Z} .
$$

Given the integer period $P$ of the diffusion coefficient we write the traveling wave equations in terms of $P$ time dependent wave speeds and $P$ time dependent wave forms. For any $j \in \mathbb{Z}$ there is a $k \in\left\{-N_{l}, \ldots, N_{r}\right\}$ and an $n \in \mathbb{Z}$ such that $j=k+P n$. Thus for all $j \in \mathbb{Z}$

$$
\alpha_{j}=\alpha_{k+n P}=\alpha_{k} \quad \text { for } \quad \text { some } \quad k=-N_{l}, \ldots, N_{r} \quad \text { and } \quad n \in \mathbb{Z},
$$


which in turn implies

$$
c_{k}(t)=c_{k+n P}(t) \quad \text { for } \quad k=-N_{l}, \ldots, N_{r} \quad \text { and } \quad n \in \mathbb{Z} .
$$

For spatially periodic diffusion we are able write the traveling wave equation (1.9) as a system of mixed differential-difference equations by defining

$$
\varphi_{k}(x)=\phi\left(x+\xi_{k}\right)
$$

for $k=-N_{l}, \ldots, N_{r}, x \in \mathbb{R}$, requiring that $\varphi_{k}(x) \in C(\mathbb{R})$. Relation (2.1) implies $\phi\left(x+\xi_{k+n P}\right)=\phi\left(x+n P+\xi_{k}\right)=\varphi_{k}(x+n P)$ and $\varphi_{k}(n P)=\phi\left(\xi_{k+n P}\right)=u_{k+n P}(t)$. The functions $\varphi_{k}$ vary as the $\xi_{k}$ vary and the $\xi_{k}$ evolve with $t$. In the following derivation we assume $t$ is fixed and define $c_{k} \equiv c_{k}(t)$ for $k=-N_{l}, \ldots, N_{r}$.

Let

$$
\underline{\varphi}(x)=\left[\varphi_{-N_{l}}(x), \varphi_{-N_{l}+1}(x), \varphi_{-N_{l}+2}(x), \ldots, \varphi_{N_{r}}(x)\right]^{T},
$$

let

$$
\underline{f_{\varphi}}(x)=\left[f\left(\varphi_{-N_{l}}(x), a\right), f\left(\varphi_{-N_{l}+1}(x), a\right), f\left(\varphi_{-N_{l}+2}(x), a\right), \ldots, f\left(\varphi_{N_{r}}(x), a\right)\right]^{T},
$$

let $\mathbf{C}=\operatorname{diag}\left\{c_{k}\right\}, k=-N_{l}, \ldots, N_{r}$, and let

$$
\begin{aligned}
& \mathbf{L}_{0}=\left[\begin{array}{ccccc}
\alpha_{-N_{l}}+\alpha_{-N_{l}+1} & -\alpha_{-N_{l}+1} & 0 & \cdots & 0 \\
-\alpha_{-N_{l}+1} & \alpha_{-N_{l}+1}+\alpha_{-N_{l}+2} & -\alpha_{-N_{l}+2} & & 0 \\
0 & -\alpha_{-N_{l}+2} & \alpha_{-N_{l}+2}+\alpha_{-N_{l}+3} & & \\
\vdots & & & \ddots & \\
0 & \ldots & -\alpha_{N_{r}-1} & \alpha_{N_{r}-1}+\alpha_{N_{r}} & -\alpha_{N_{r}} \\
0 & \cdots & 0 & -\alpha_{N_{r}} & \alpha_{N_{r}}+\alpha_{-N_{l}}
\end{array}\right] \\
& \mathbf{L}_{+}=\left[\begin{array}{ccccc}
0 & 0 & 0 & \cdots & \alpha_{-N_{l}} \\
0 & 0 & 0 & & 0 \\
0 & 0 & 0 & & \\
\vdots & & & \ddots & \\
& & 0 & 0 & 0 \\
0 & \cdots & 0 & 0 & 0
\end{array}\right], \quad \mathbf{L}_{-}=\left[\begin{array}{ccccc}
0 & 0 & 0 & \cdots & 0 \\
0 & 0 & 0 & & 0 \\
0 & 0 & 0 & & \\
\vdots & & & \ddots & \\
& & 0 & 0 & 0 \\
\alpha_{-N_{l}} & \cdots & 0 & 0 & 0
\end{array}\right]
\end{aligned}
$$

The system of equations in $\varphi_{k}$ that we need to solve to obtain the solution to (1.9) can be represented by the finite system of equations

$$
-\mathbf{C} \underline{\varphi}^{\prime}(x)=\mathbf{L}_{-} \underline{\varphi}(x+P)-\mathbf{L}_{0} \underline{\varphi}(x)+\mathbf{L}_{+} \underline{\varphi}(x-P)-\underline{f_{\varphi}}(x) .
$$

Observe that the delay terms for this system no longer depend on time or lattice position. For fixed $t$ the solution vector $\underline{\varphi}$ is translationally invariant. We choose a particular translate by specifying the phase condition $\phi(0)=a$. This produces, by (2.1), the set of phase conditions

$$
\varphi_{k}\left(-\xi_{k}\right)=a \quad \text { for } \quad k=-N_{l}, \ldots, N_{r},
$$

which we apply to system (2.2). This choice of phase conditions defines a set of phase shifts between the curves $\varphi_{k}$ and allows those phase shifts to change with time. If 
we assume that the function $\varphi(x)$ is strictly increasing in $x$, then this choice of phase condition implies

$$
\varphi_{k}(x)<a \text { when } \quad x<-\xi_{k} \quad \text { and } \quad \varphi_{k}(x)>a \text { when } \quad x>-\xi_{k}
$$

for $k=-N_{l}, \ldots, N_{r}$.

Since we are looking for traveling wave solutions, solutions which connect the stable equilibria of $\dot{u}=f(u)$, and since $\varphi_{k}(x)=\varphi\left(x+\xi_{k}\right)$, it is natural to impose the boundary conditions

$$
\varphi_{k}(-\infty)=0, \quad \varphi_{k}(\infty)=1
$$

for $k=-N_{l}, \ldots, N_{r}$.

3. An analytic solution. We now derive the solution of the traveling wave equation (1.9) with the periodic diffusion coefficients $\alpha_{k}$, the boundary conditions (2.4), and the piecewise linear function $f_{1}(1.5)$. The inequalities in (2.3) imply, with $h$ defined as the Heaviside step function

$$
h(y)= \begin{cases}0 & y<0 \\ 1 & y>0\end{cases}
$$

that

$$
h\left(\varphi_{k}(x)-a\right)=h\left(x+\xi_{k}\right) \quad \text { for } \quad x \neq-\xi_{k} \quad \text { and } \quad k=-N_{l}, \ldots, N_{r} .
$$

We rewrite the nonlinearity $f_{1}$ as the set of nonlinearities

$$
f_{1}\left(\varphi_{k}(x), a\right)=\varphi_{k}(x)-h\left(\varphi_{k}(x)-a\right)=\varphi_{k}(x)-h_{k}(x) \quad \text { for } \quad k=-N_{l}, \ldots, N_{r},
$$

where $h_{k}(x)=h\left(x+\xi_{k}\right)$. For $\varphi_{k}(x)$ continuous, the jump discontinuity in $f_{1}\left(\varphi_{k}(x)\right)$ at $x=-\xi_{k}$ produces a jump discontinuity in $\varphi_{k}^{\prime}(x)$ at $x=-\xi_{k}$, for $k=-N_{l}, \ldots, N_{r}$.

Our derivation focuses on the construction of the solutions, $\varphi_{k}(x), k=-N_{l}, \ldots, N_{r}$, for (2.2). We begin by defining the functions $\phi_{k}(x)=e^{-\varepsilon x} \varphi_{k}(x)$ for $k=-N_{l}, \ldots, N_{r}$ and substituting them into the system (2.2). The solutions to the resulting system asymptotically approach zero as $|x| \rightarrow \infty$. This and the linear nature of $f_{1}$ allows us to apply the Fourier transform. After some manipulation, we then apply the Fourier inversion theorem to obtain $\varphi_{k}(x)=e^{\varepsilon x} \phi_{k}(x)$.

3.1. Change of variables. We begin our derivation of a solution with a preliminary lemma.

Lemma 3.1. Let $\varphi_{k}, k=-N_{l}, \ldots, N_{r}$ be defined as in (2.1). Then there exists an $\varepsilon_{0}>0$ such that, for some $K>0$,

$$
\left|\varphi_{k}(x)\right| \leq K e^{\varepsilon_{0} x} \quad \text { for } \quad x \leq-N_{r} .
$$

Proof. Lemma 4.1 in [5] (see also Lemma 2.1 in [8]) implies that for each $\varphi_{k}$, $k=-N_{l}, \ldots, N_{r}$, there exists an $\varepsilon_{k 0}>0$ such that, for some $K_{k}>0, \quad\left|\varphi_{k}(x)\right| \leq$ $K_{k} e^{\varepsilon_{k 0} x}$ for $x \leq-N_{r}$. Let

$$
K=\max _{k=-N_{l}, \ldots, N_{r}} K_{k} \quad \text { and } \quad \varepsilon_{0}=\min _{k=-N_{l}, \ldots, N_{r}} \varepsilon_{k 0} .
$$

Let $\phi_{k}(x)=e^{-\varepsilon x} \varphi_{k}(x)$ for $k=-N_{l}, \ldots, N_{r}$ with $\varepsilon>0, \varepsilon$ small. By Lemma 3.1, if $\varphi_{k}, k=-N_{l}, \ldots, N_{r}$, is a solution element to (1.9), then, as $x \rightarrow-\infty$,

$$
\phi_{k}(x)=e^{-\varepsilon x} \varphi_{k}(x) \leq\left|e^{-\varepsilon x}\right|\left|\varphi_{k}(x)\right| \leq\left|K e^{\left(\varepsilon_{0}-\varepsilon\right) x}\right| \rightarrow 0 \text { for } 0<\varepsilon<\varepsilon_{0} .
$$


Also as $x \rightarrow \infty$,

$$
\phi_{k}(x)=e^{-\varepsilon x} \varphi_{k}(x) \rightarrow 0
$$

since $\varphi_{k}(+\infty)=1$ and $\varepsilon_{k}>0$. This implies that $\phi_{k}(-\infty)=0$ and $\phi_{k}(+\infty)=0$. Substituting $\varphi_{k}(x)=e^{\varepsilon x} \phi_{k}(x), k=-N_{l}, \ldots, N_{r}$, into (2.2) and simplifying, we obtain

$$
-{ }_{-} \underline{\phi}^{\prime}(x)=\left[e^{-P \varepsilon} \mathbf{L}_{-} \underline{\phi}(x-P)-\mathbf{L}_{0} \underline{\phi}(x)+e^{P \varepsilon} \mathbf{L}_{+} \underline{\phi}(x+P)\right]-[\mathbf{I}-\varepsilon \mathbf{C}(t)] \underline{\phi}(x)+e^{-\varepsilon x} \underline{h}(x),
$$

where

$$
\underline{h}(x)=\left[h_{-N_{l}}(x), h_{-N_{l}+1}(x), h_{-N_{l}+2}(x), \ldots, h_{N_{r}}(x)\right]^{T} .
$$

3.2. Fourier transform. We now apply the Fourier transforms

$$
\hat{\phi}_{k}(s)=\int_{-\infty}^{\infty} e^{-i s x} \phi_{k}(x) d x, \quad k=-N_{l}, \ldots, N_{r},
$$

where $\varepsilon>0$ is sufficiently small, to both sides of (3.2). Then

$$
\begin{gathered}
\hat{\phi}_{-N_{l}}(s)=\int_{-\infty}^{\infty} e^{-i s x} \phi_{-N_{l}}(x) d x=-\frac{i}{s} \int_{-\infty}^{\infty} e^{-i s x} \phi_{-N_{l}}^{\prime}(x) d x \\
=\frac{i}{s c_{-N_{l}}} \int_{-\infty}^{\infty}\left[\alpha_{-N_{l}+1} e^{-i s x} \phi_{-N_{l}+1}(x)-\alpha_{-N_{l}+1} e^{-i s x} \phi_{-N_{l}}(x)\right. \\
+\alpha_{-N_{l}} e^{-i s x} e^{-P \varepsilon} \phi_{N_{r}}(x-P) \\
-\alpha_{-N_{l}} e^{-i s x} \phi_{-N_{l}}(x)-\left(1-c_{-N_{l}} \varepsilon\right) e^{-i s x} \phi_{-N_{l}}(x) \\
\left.+e^{-(i s+\varepsilon) x} h\left(x+\xi_{-N_{l}}\right)\right] d x, \\
\hat{\phi}_{m}(s)=\frac{i}{s c_{m}} \int_{-\infty}^{\infty}\left[\alpha_{m+1} e^{-i s x} \phi_{m+1}(x)-\alpha_{m+1} e^{-i s x} \phi_{m}(x)+\alpha_{m} e^{-i s x} \phi_{m-1}(x)\right. \\
\left.-\alpha_{m} e^{-i s x} \phi_{m}(x)-\left(1-c_{m} \varepsilon\right) e^{-i s x} \phi_{m}(x)+e^{-(i s+\varepsilon) x} h\left(x+\xi_{m}\right)\right] d x, \\
\hat{\phi}_{N_{r}}(s)=\int_{-\infty}\left[\alpha_{-N_{l}} e^{-i s x} e^{P \varepsilon} \phi_{-N_{l}}(x+P)-\alpha{ }_{-N_{l}} e^{-i s x} \phi_{N_{r}}(x)+\alpha_{N_{r}} e^{-i s x} \phi_{N_{r}-1}(x)\right. \\
\left.\frac{i}{s c_{N_{r}}} \int_{N_{r}} e^{-i s x} \phi_{N_{r}}(x)-\left(1-c_{N_{r}} \varepsilon\right) e^{-i s x} \phi_{N_{r}}(x)+e^{-(i s+\varepsilon) x} h\left(x+\xi_{N_{r}}\right)\right] d x \\
(3.3) \\
\text { for } m=-N_{l}+1, \ldots, N_{r}-1 .
\end{gathered}
$$

3.3. Solving in Fourier space. Let $A_{k}=\left(-c_{k}(i s+\varepsilon)+\alpha_{k+1}+\alpha_{k}+1\right)$, $k=-N_{l}, \ldots, N_{r}$, where $\alpha_{N_{r}+1}=\alpha_{-N_{l}}$, let

$$
\hat{\phi}(s)=\left[\hat{\phi}_{-N_{l}}(s), \hat{\phi}_{-N_{l}+1}(s), \ldots, \hat{\phi}_{N_{r}}(s)\right]^{T},
$$


let

$$
\underline{v}=\left[\frac{e^{(i s+\varepsilon)\left(\xi_{-N_{l}}\right)}}{i s+\varepsilon}, \frac{e^{(i s+\varepsilon)\left(\xi_{-N_{l}+1}\right)}}{i s+\varepsilon}, \ldots, \frac{e^{(i s+\varepsilon)\left(\xi_{N_{r}}\right)}}{i s+\varepsilon}\right]^{T}
$$

and let

$$
\mathbf{M}=\left[\begin{array}{ccccc}
A_{-N_{l}} & -\alpha_{-N_{l}+1} & 0 & \cdots & -\alpha_{-N_{l}} e^{-P(i s+\varepsilon)} \\
-\alpha_{-N_{l}+1} & A_{-N_{l}+1} & -\alpha_{-N_{l}+2} & & 0 \\
0 & -\alpha_{-N_{l}+2} & A_{-N_{l}+2} & & \\
\vdots & & & \ddots & \\
-\alpha_{-N_{l}} e^{P(i s+\varepsilon)} & \ldots & -\alpha_{N_{r}-1} & A_{N_{r}-1} & -\alpha_{N_{r}}
\end{array}\right] .
$$

Then after performing the integrations, system (3.3) can be written as

$$
\mathbf{M} \underline{\hat{\phi}}=\underline{v} .
$$

Remark 3.1. The translation of system (2.2) into Fourier space contains no delay terms.

Observe that the matrix $M$ is irreducibly diagonally dominant and hence invertible (see Theorem 2.3.8 in [21]). We obtain $\varphi_{k}(x)$ by applying the inverse Fourier transform

$$
\varphi_{k}(x)=e^{\varepsilon x} \phi_{k}(x)=\frac{1}{2 \pi} \int_{-\infty}^{\infty} e^{(i s+\varepsilon) x} \hat{\phi}_{k}(s) d s \quad \text { for } \quad k=-N_{l}, \ldots, N_{r},
$$

which is absolutely convergent. Although in theory this appears simple enough, we present the case for $P=2$ to demonstrate important features of the solutions of spatially discrete reaction-diffusion equation with periodic diffusion.

4. Example: Alternating diffusion coefficient. Consider a diffusion coefficient function of period $P=2$ defined as

$$
\alpha_{j}=\left\{\begin{array}{lll}
\alpha_{e} & j & \text { even } \\
\alpha_{o} & j & \text { odd }
\end{array}\right.
$$

with $\alpha_{e}, \alpha_{o} \in \mathbb{R}^{+}$. Let $c_{e}(t)=c_{j}(t), j$ even, $c_{o}(t)=c_{j}(t), j$ odd. Define

$$
\varphi_{e}(x)=\varphi\left(x-\int_{0}^{t} c_{e}(s) d s\right), \quad \varphi_{o}(x)=\varphi\left(x+1-\int_{0}^{t} c_{o}(s) d s\right) .
$$

We obtain the system

$$
\begin{aligned}
& -c_{e}(t) \varphi_{e}^{\prime}(x)=\alpha_{o}\left(\varphi_{o}(x)-\varphi_{e}(x)\right)+\alpha_{e}\left(\varphi_{o}(x-2)-\varphi_{e}(x)\right)-f_{1}\left(\varphi_{e}(x)\right) \\
& -c_{o}(t) \varphi_{o}^{\prime}(x)=\alpha_{e}\left(\varphi_{e}(x+2)-\varphi_{o}(x)\right)+\alpha_{o}\left(\varphi_{e}(x)-\varphi_{o}(x)\right)-f_{1}\left(\varphi_{o}(x)\right) .
\end{aligned}
$$

The phase condition $\phi(0)=a$ implies $\varphi_{e}\left(\int_{0}^{t} c_{e}(s) d s\right)=a$ and $\varphi_{o}\left(-1+\int_{0}^{t} c_{o}(s) d s\right)=a$. The nonlinearities are $f_{1}\left(\varphi_{e}, a\right)=\varphi_{e}-h\left(x-\int_{0}^{t} c_{e}(s) d s\right), f_{1}\left(\varphi_{o}, a\right)=\varphi_{o}-h(x+1-$ $\left.\int_{0}^{t} c_{o}(s) d s\right)$. 
4.1. An analytic solution. We now derive the solution of the traveling wave equation (1.9) by deriving the solution for the pair of traveling wave equations (4.3) with the boundary conditions (2.4) and the piecewise linear function $f_{1},(1.5)$. From our derived solution, we obtain a relationship between the detuning parameter, $a$, and the wave speeds, $c_{e}$ and $c_{o}$.

This section begins with the construction of solutions, $\varphi_{e}(x)$ and $\varphi_{o}(x)$, for (1.9). The construction consists of defining functions $\phi_{e}(x)=e^{-\varepsilon x} \varphi_{e}(x)$ and $\phi_{o}(x)=$ $e^{-\varepsilon x} \varphi_{o}(x)$ and transforming (4.3). To the $\phi_{e}(x), \phi_{o}(x)$ versions of the equations, we apply the Fourier transform, and after some manipulation, we derive a formula for $\varphi_{e}(x)$ and $\varphi_{o}(x)$.

Fix $t$ and let $c_{e} \equiv c_{e}(t), c_{o} \equiv c_{o}(t), r_{e} \equiv \int_{0}^{t} c_{e}(s) d s$, and $r_{o} \equiv \int_{0}^{t} c_{o}(s) d s$, thus $\xi_{e}=-r_{e}$ and $\xi_{o}=1-r_{o}$. Using the derivation from the general period derivation, we obtain

$$
\left(-c_{e}(i s+\varepsilon)+\alpha_{o}+\alpha_{e}+1\right) \hat{\phi}_{e}(s)-\left(\alpha_{o}+\alpha_{e} e^{-2(i s+\varepsilon)}\right) \hat{\phi}_{o}(s)=\frac{e^{-(i s+\varepsilon) r_{e}}}{i s+\varepsilon},
$$

or

$$
\left[\begin{array}{cc}
A_{e} & -\alpha_{o}-\alpha_{e} e^{-2(i s+\varepsilon)} \\
-\alpha_{o}-\alpha_{e} e^{2(i s+\varepsilon)} & A_{o}
\end{array}\right]\left[\begin{array}{c}
\hat{\phi}_{e} \\
\hat{\phi}_{o}
\end{array}\right]=\left[\begin{array}{c}
\frac{e^{-(i s+\varepsilon) r_{e}}}{i s+\varepsilon} \\
\frac{e^{(i s+\varepsilon)\left(1-r_{o}\right)}}{i s+\varepsilon}
\end{array}\right],
$$

with $A_{e}=-c_{e}(i s+\varepsilon)+\alpha_{o}+\alpha_{e}+1$ and $A_{o}=-c_{o}(i s+\varepsilon)+\alpha_{o}+\alpha_{e}+1$. This is a special case of the matrix M. Solving, we obtain

$$
\hat{\phi}_{e}(s)=\frac{P(s-i \varepsilon)}{(i s+\varepsilon) R(s-i \varepsilon)}, \quad \hat{\phi}_{o}(s)=\frac{Q(s-i \varepsilon)}{(i s+\varepsilon) R(s-i \varepsilon)},
$$

where

$$
\begin{aligned}
& P(q)=e^{-i q r_{e}}\left[-i c_{o} q+\alpha_{o}+\alpha_{e}+1\right]+e^{-i q r_{o}}\left[\alpha_{o} e^{i q}+\alpha_{e} e^{-i q}\right] \\
& Q(q)=e^{i q\left(1-r_{o}\right)}\left[-i c_{e} q+\alpha_{o}+\alpha_{e}+1\right]+e^{i q\left(1-r_{e}\right)}\left[\alpha_{e} e^{i q}+\alpha_{o} e^{-i q}\right] \\
& R(q)=-i\left(c_{e}+c_{o}\right) q\left(\alpha_{o}+\alpha_{e}+1\right)+2 \alpha_{o}+2 \alpha_{e}+1+2 \alpha_{o} \alpha_{e}(1-\cos 2 q)-c_{e} c_{o} q^{2} .
\end{aligned}
$$

The following lemma is a simple extension of Lemma 4.4 in [5] and is based on the characteristic equation of the system.

Lemma 4.1. For $c_{e}, c_{o} \neq 0$, the function $R$ in (4.6) possesses a simple root $q=-i z_{0}$ with $z_{0}>0$. Moreover, there exists an $\epsilon_{0}>0$ such that if $q \in \mathbb{C}$ is any other root of $R$ satisfying $\operatorname{Im} q \leq 0$, then in fact $\operatorname{Im} q<-z_{0}-\epsilon_{0}$.

We now use the Fourier inversion theorem which gives

$$
\begin{aligned}
& \varphi_{e}(x)=e^{\varepsilon x} \phi_{e}(x)=\frac{1}{2 \pi} \int_{-\infty}^{+\infty} e^{(i s+\varepsilon) x} \hat{\phi}_{e}(s) d s, \\
& \varphi_{o}(x)=e^{\varepsilon x} \phi_{o}(x)=\frac{1}{2 \pi} \int_{-\infty}^{+\infty} e^{(i s+\varepsilon) x} \hat{\phi}_{o}(s) d s,
\end{aligned}
$$

which are absolutely convergent. This implies

$$
\begin{aligned}
& \varphi_{e}(x)=\frac{1}{2 \pi} \int_{-\infty}^{+\infty} \frac{e^{(i s+\varepsilon) x} P(s-i \varepsilon)}{(i s+\varepsilon) R(s-i \varepsilon)} d s=\frac{1}{2 \pi i} \int_{-i \varepsilon-\infty}^{-i \varepsilon+\infty} \frac{e^{i s x} P(s)}{s R(s)} d s \\
& \varphi_{o}(x)=\frac{1}{2 \pi} \int_{-\infty}^{+\infty} \frac{e^{(i s+\varepsilon) x} Q(s-i \varepsilon)}{(i s+\varepsilon) R(s-i \varepsilon)} d s=\frac{1}{2 \pi i} \int_{-i \varepsilon-\infty}^{-i \varepsilon+\infty} \frac{e^{i s x} Q(s)}{s R(s)} d s .
\end{aligned}
$$


Because of the simple pole at $s=0$, we break up the integrals into

$$
\begin{aligned}
& \varphi_{e}(x)=\frac{1}{2 \pi i}\left(\int_{C_{\varepsilon}}+\int_{S_{\varepsilon}}\right) \frac{e^{i s x} P(s)}{s R(s)} d s, \\
& \varphi_{o}(x)=\frac{1}{2 \pi i}\left(\int_{C_{\varepsilon}}+\int_{S_{\varepsilon}}\right) \frac{e^{i s x} Q(s)}{s R(s)} d s,
\end{aligned}
$$

where $C_{\varepsilon}$ is $(-\infty,-\varepsilon]$ and $[+\varepsilon,+\infty)$ on the real axis, and $S_{\varepsilon}$ is the half-circle at the origin. First consider the $C_{\varepsilon}$ integrals. We have

$$
\begin{aligned}
\frac{1}{2 \pi i} \int_{C_{\varepsilon}} \frac{e^{i s x} P(s)}{s R(s)} d s= & \frac{1}{2 \pi i}\left(\int_{-\infty}^{-\varepsilon}+\int_{+\varepsilon}^{+\infty}\right) \frac{e^{i s x} P(s)}{s R(s)} d s \\
= & \frac{1}{2 \pi i} \int_{+\varepsilon}^{+\infty}\left[\frac{e^{i s x} P(s)}{s R(s)}-\frac{e^{-i s x} P(-s)}{s R(-s)}\right] d s \\
= & \frac{1}{2 \pi i} \int_{+\varepsilon}^{+\infty} \frac{e^{i s x} P(s) R(-s)-e^{-i s x} P(-s) R(s)}{s R(s) R(-s)} d s \\
= & \frac{1}{\pi} \int_{+\varepsilon}^{+\infty} \frac{A(s)\left[-s c_{o} \cos s\left(x-r_{e}\right)+\left(\alpha_{o}+\alpha_{e}+1\right) \sin s\left(x-r_{e}\right)\right]}{s\left(A^{2}(s)+B^{2}(s)\right)} d s \\
& +\frac{1}{\pi} \int_{+\varepsilon}^{+\infty} \frac{B(s)\left[s c_{o} \sin s\left(x-r_{e}\right)+\left(\alpha_{o}+\alpha_{e}+1\right) \cos s\left(x-r_{e}\right)\right]}{s\left(A^{2}(s)+B^{2}(s)\right)} d s \\
& +\frac{1}{\pi} \int_{+\varepsilon}^{+\infty} \frac{A(s)\left[\alpha_{o} \sin s\left(x+1-r_{o}\right)+\alpha_{e} \sin s\left(x-1-r_{o}\right)\right]}{s\left(A^{2}(s)+B^{2}(s)\right)} d s \\
& +\frac{1}{\pi} \int_{+\varepsilon}^{+\infty} \frac{B(s)\left[\alpha_{o} \cos s\left(x+1-r_{o}\right)+\alpha_{e} \cos s\left(x-1-r_{o}\right)\right]}{s\left(A^{2}(s)+B^{2}(s)\right)} d s
\end{aligned}
$$

and

$$
\begin{aligned}
\frac{1}{2 \pi i} \int_{C_{\varepsilon}} \frac{e^{i s x} Q(s)}{s R(s)} d s \\
=\frac{1}{2 \pi i}\left(\int_{-\infty}^{-\varepsilon}+\int_{+\varepsilon}^{+\infty}\right) \frac{e^{i s x} Q(s)}{s R(s)} d s \\
=\frac{1}{\pi} \int_{+\varepsilon}^{+\infty} \frac{A(s)\left[-s c_{e} \cos s\left(x+1-r_{o}\right)+\left(\alpha_{o}+\alpha_{e}+1\right) \sin s\left(x+1-r_{o}\right)\right]}{s\left(A^{2}(s)+B^{2}(s)\right)} d s \\
+\frac{1}{\pi} \int_{+\varepsilon}^{+\infty} \frac{B(s)\left[s c_{e} \sin s\left(x+1-r_{o}\right)+\left(\alpha_{o}+\alpha_{e}+1\right) \cos s\left(x+1-r_{o}\right)\right]}{s\left(A^{2}(s)+B^{2}(s)\right)} d s \\
+\frac{1}{\pi} \int_{+\varepsilon}^{+\infty} \frac{A(s)\left[\alpha_{e} \sin s\left(x+2-r_{e}\right)+\alpha_{o} \sin s\left(x-r_{e}\right)\right]}{s\left(A^{2}(s)+B^{2}(s)\right)} d s \\
+\frac{1}{\pi} \int_{+\varepsilon}^{+\infty} \frac{B(s)\left[\alpha_{e} \cos s\left(x+2-r_{e}\right)+\alpha_{o} \cos s\left(x-r_{e}\right)\right]}{s\left(A^{2}(s)+B^{2}(s)\right)} d s,
\end{aligned}
$$

where

$$
\begin{aligned}
& A(s)=1-c_{e} c_{o} s^{2}+2 \alpha_{e} \alpha_{o}(1-\cos (2 s))+2\left(\alpha_{e}+\alpha_{o}\right), \\
& B(s)=\left(c_{e}+c_{o}\right) s\left(\alpha_{e}+\alpha_{o}+1\right) .
\end{aligned}
$$


For $s \in \mathbb{R}, A(s)=\operatorname{Re}[R(s)]$ and $B(s)=-\operatorname{Im}[R(s)]$.

For the $S_{\varepsilon}$ integrals, we have

$$
\begin{aligned}
& \frac{1}{2 \pi i} \int_{S_{\varepsilon}} \frac{e^{i s \xi} P(s)}{s R(s)} d s=\frac{1}{2 \pi i} 2 \pi i \frac{1}{2} \operatorname{Res}\left(\frac{e^{i s \xi} P(s)}{s R(s)}, 0\right)=\frac{1}{2}, \\
& \frac{1}{2 \pi i} \int_{S_{\varepsilon}} \frac{e^{i s \xi} Q(s)}{s R(s)} d s=\frac{1}{2} .
\end{aligned}
$$

Combining the integral results (4.8) and (4.9) in (4.7), and letting $\varepsilon \rightarrow 0$, we obtain an explicit formula for $\varphi_{e}(x)$ and $\varphi_{o}(x)$. From (4.7), we have the following formula for the solution of (4.3) with (4.1) for $c_{e}, c_{o} \neq 0$ :

$$
\begin{aligned}
\varphi_{e}(x)=\frac{1}{2} & +\frac{1}{\pi} \int_{0}^{\infty} \frac{A(s)\left[-s c_{o} \cos s\left(x-r_{e}\right)+\left(\alpha_{o}+\alpha_{e}+1\right) \sin s\left(x-r_{e}\right)\right]}{s\left(A^{2}(s)+B^{2}(s)\right)} d s \\
& +\frac{1}{\pi} \int_{0}^{\infty} \frac{B(s)\left[s c_{o} \sin s\left(x-r_{e}\right)+\left(\alpha_{o}+\alpha_{e}+1\right) \cos s\left(x-r_{e}\right)\right]}{s\left(A^{2}(s)+B^{2}(s)\right)} d s \\
& +\frac{1}{\pi} \int_{0}^{\infty} \frac{A(s)\left[\alpha_{o} \sin s\left(x+1-r_{o}\right)+\alpha_{e} \sin s\left(x-1-r_{o}\right)\right]}{s\left(A^{2}(s)+B^{2}(s)\right)} d s \\
& +\frac{1}{\pi} \int_{0}^{\infty} \frac{B(s)\left[\alpha_{o} \cos s\left(\xi+1-r_{o}\right)+\alpha_{e} \cos s\left(\xi-1-r_{o}\right)\right]}{s\left(A^{2}(s)+B^{2}(s)\right)} d s,
\end{aligned}
$$

and

$$
\begin{aligned}
& \varphi_{o}(x)=\frac{1}{2}+\frac{1}{\pi} \int_{0}^{\infty} \frac{A(s)\left[-s c_{e} \cos s\left(x+1-r_{o}\right)+\left(\alpha_{o}+\alpha_{e}+1\right) \sin s\left(x+1-r_{o}\right)\right]}{s\left(A^{2}(s)+B^{2}(s)\right)} d s \\
&+\frac{1}{\pi} \int_{0}^{\infty} \frac{B(s)\left[s c_{e} \sin s\left(x+1-r_{o}\right)+\left(\alpha_{o}+\alpha_{e}+1\right) \cos s\left(x+1-r_{o}\right)\right]}{s\left(A^{2}(s)+B^{2}(s)\right)} d s \\
&+\frac{1}{\pi} \int_{0}^{\infty} \frac{A(s)\left[\alpha_{e} \sin s\left(x+2-r_{e}\right)+\alpha_{o} \sin s\left(x-r_{e}\right)\right]}{s\left(A^{2}(s)+B^{2}(s)\right)} d s \\
&(4.10) \quad+\frac{1}{\pi} \int_{0}^{\infty} \frac{B(s)\left[\alpha_{e} \cos s\left(x+2-r_{e}\right)+\alpha_{o} \cos s\left(x-r_{e}\right)\right]}{s\left(A^{2}(s)+B^{2}(s)\right)} d s .
\end{aligned}
$$

Remark 4.1. Let $\varphi_{e}$ and $\varphi_{o}$ be considered as functions of $r_{e}=\int_{0}^{t} c_{e}(q) d q$ and $r_{o}=\int_{0}^{t} c_{o}(q) d q$, i.e., $\varphi_{e}\left(x, r_{e}, r_{o}\right)$ and $\varphi_{o}\left(x, r_{e}, r_{o}\right)$.

(i) Observing that

$$
\begin{aligned}
1-\varphi_{e}(- & \left.-1,-r_{o},-r_{e}\right) \\
=\frac{1}{2} & -\frac{1}{\pi} \int_{0}^{\infty} \frac{A(s)\left[s c_{e} \cos s\left(-x-1+r_{o}\right)+\left(\alpha_{o}+\alpha_{e}+1\right) \sin s\left(-x-1+r_{o}\right)\right]}{s\left(A^{2}(s)+B^{2}(s)\right)} d s \\
& -\frac{1}{\pi} \int_{0}^{\infty} \frac{B(s)\left[-s c_{e} \sin s\left(-x-1+r_{o}\right)-\left(\alpha_{o}+\alpha_{e}+1\right) \cos s\left(-x-1+r_{e}\right)\right]}{s\left(A^{2}(s)+B^{2}(s)\right)} d s \\
& -\frac{1}{\pi} \int_{0}^{\infty} \frac{A(s)\left[\alpha_{o} \sin s\left(-x+r_{e}\right)+\alpha_{e} \sin s\left(-x-2+r_{e}\right)\right]}{s\left(A^{2}(s)+B^{2}(s)\right)} d s \\
& -\frac{1}{\pi} \int_{0}^{\infty} \frac{B(s)\left[\alpha_{o} \cos s\left(-x+r_{e}\right)+\alpha_{e} \cos s\left(-x-2+r_{e}\right)\right]}{s\left(A^{2}(s)+B^{2}(s)\right)} d s \\
= & \varphi_{o}\left(x, r_{e}, r_{o}\right),
\end{aligned}
$$


and

$$
\begin{aligned}
1- & \varphi_{o}\left(-x-1,-r_{o},-r_{e}\right) \\
= & \frac{1}{2}-\frac{1}{\pi} \int_{0}^{\infty} \frac{A(s)\left[s c_{o} \cos s\left(-x+r_{e}\right)+\left(\alpha_{o}+\alpha_{e}+1\right) \sin s\left(-x+r_{e}\right)\right]}{s\left(A^{2}(s)+B^{2}(s)\right)} d s \\
& -\frac{1}{\pi} \int_{0}^{\infty} \frac{B(s)\left[-s c_{e} \sin s\left(-x+r_{e}\right)+\left(\alpha_{o}+\alpha_{e}+1\right) \cos s\left(-x+r_{e}\right)\right]}{s\left(A^{2}(s)+B^{2}(s)\right)} d s \\
& -\frac{1}{\pi} \int_{0}^{\infty} \frac{A(s)\left[\alpha_{e} \sin s\left(-x+1+r_{o}\right)+\alpha_{o} \sin s\left(-x-1+r_{o}\right)\right]}{s\left(A^{2}(s)+B^{2}(s)\right)} d s \\
& -\frac{1}{\pi} \int_{0}^{\infty} \frac{B(s)\left[\alpha_{e} \cos s\left(-x+1+r_{o}\right)+\alpha_{o} \cos s\left(-x-1+r_{o}\right)\right]}{s\left(A^{2}(s)+B^{2}(s)\right)} d s \\
= & \varphi_{e}\left(x, r_{e}, r_{o}\right),
\end{aligned}
$$

we have the symmetry property $1-\varphi_{e}\left(-x-1,-r_{o},-r_{e}\right)=\varphi_{o}\left(x, r_{e}, r_{o}\right)$, and $1-$ $\varphi_{o}\left(-x-1,-r_{o},-r_{e}\right)=\varphi_{e}\left(x, r_{e}, r_{o}\right)$.

(ii) Recalling that

$$
\varphi_{e}(x)=\frac{1}{2 \pi i} \int_{-i \varepsilon-\infty}^{-i \varepsilon+\infty} \frac{e^{i s x} P(s)}{s R(s)} d s \quad \text { and } \quad \varphi_{o}(x)=\frac{1}{2 \pi i} \int_{-i \varepsilon-\infty}^{-i \varepsilon+\infty} \frac{e^{i s x} Q(s)}{s R(s)} d s,
$$

we can see that as $x \rightarrow-\infty, \varphi_{e}(x) \rightarrow 0$ and $\varphi_{o}(x) \rightarrow 0$. Our symmetry property then gives us that $\varphi_{e}(+\infty)=1$ and $\varphi_{o}(+\infty)=1$. This confirms our choice of boundary conditions.

Remark 4.2. (i) As discussed before, $\varphi(\xi)=a$ for only one value of $\xi$. Let $\xi=0$. This then gives us the following relation between $a$ and $\left(c_{e}, c_{o}\right)$ :

$$
\begin{aligned}
a= & \varphi_{e}\left(r_{e}\right) \\
= & \frac{1}{2}+\frac{1}{\pi} \int_{0}^{\infty} \frac{-s c_{o} A(s)+\left[\alpha_{o}+\alpha_{e}+1\right] B(s)}{s\left(A^{2}(s)+B^{2}(s)\right)} d s \\
& +\frac{1}{\pi} \int_{0}^{\infty} \frac{A(s)\left[\left(\alpha_{o}-\alpha_{e}\right) \sin (s) \cos s\left(r_{e}-r_{o}\right)+\left(\alpha_{o}+\alpha_{e}\right) \cos (s) \sin s\left(r_{e}-r_{o}\right)\right]}{s\left(A^{2}(s)+B^{2}(s)\right)} d s \\
& +\frac{1}{\pi} \int_{0}^{\infty} \frac{B(s)\left[\left(\alpha_{o}+\alpha_{e}\right) \cos (s) \cos s\left(r_{e}-r_{o}\right)+\left(\alpha_{e}-\alpha_{o}\right) \sin (s) \sin s\left(r_{e}-r_{o}\right)\right]}{s\left(A^{2}(s)+B^{2}(s)\right)} d s
\end{aligned}
$$

and

$$
\begin{aligned}
a= & \varphi_{o}\left(-1+r_{o}\right) \\
= & \frac{1}{2}+\frac{1}{\pi} \int_{0}^{\infty} \frac{-s c_{e} A(s)+\left[\alpha_{o}+\alpha_{e}+1\right] B(s)}{s\left(A^{2}(s)+B^{2}(s)\right)} d s \\
& +\frac{1}{\pi} \int_{0}^{\infty} \frac{A(s)\left[\left(\alpha_{e}-\alpha_{o}\right) \sin (s) \cos s\left(r_{o}-r_{e}\right)+\left(\alpha_{o}+\alpha_{e}\right) \cos (s) \sin s\left(r_{o}-r_{e}\right)\right]}{s\left(A^{2}(s)+B^{2}(s)\right)} d s \\
& +\frac{1}{\pi} \int_{0}^{\infty} \frac{B(s)\left[\left(\alpha_{o}+\alpha_{e}\right) \cos (s) \cos s\left(r_{o}-r_{e}\right)+\left(\alpha_{o}-\alpha_{e}\right) \sin (s) \sin s\left(r_{o}-r_{e}\right)\right]}{s\left(A^{2}(s)+B^{2}(s)\right)} d s
\end{aligned}
$$


when $c_{e}, c_{o} \neq 0$. Adding and subtracting (4.11) and (4.12) we obtain, for $c_{e}, c_{o} \neq 0$,

$$
\begin{aligned}
a & =\frac{1}{2}+\frac{1}{\pi} \\
& \cdot \int_{0}^{\infty} \frac{-s\left[c_{e}+c_{o}\right] A(s)+2\left[\alpha_{o}\left\{1+\cos s\left(1+r_{e}-r_{o}\right)\right\}+\alpha_{e}\left\{1+\cos s\left(1+r_{o}-r_{e}\right)\right\}+1\right] B(s)}{2 s\left(A^{2}(s)+B^{2}(s)\right)} d s,
\end{aligned}
$$

subject to the condition that

$$
\int_{0}^{\infty} \frac{\left[s\left(c_{e}-c_{o}\right)+2 \alpha_{o} \sin s\left(1+r_{e}-r_{o}\right)-2 \alpha_{e} \sin s\left(1+r_{o}-r_{e}\right)\right] A(s)}{s\left(A^{2}(s)+B^{2}(s)\right)} d s=0 .
$$

(ii) When $c_{e}=c_{o}=0$,

$$
\varphi(0)=a=\frac{1}{2} \text { and } \int_{0}^{\infty} \frac{\alpha_{o} \sin s\left(1+r_{e}-r_{o}\right)-\alpha_{e} \sin s\left(1+r_{o}-r_{e}\right)}{s A(s)} d s=0
$$

when $c_{e}=c_{o}=0$.

Remark 4.3. (i) The condition (4.14) insures that the detuning parameter $a$ is a constant value and does not vary with $x$.

(ii) The justification of the assumption that $\varphi_{e}(x)$ and $\varphi_{o}(x)$ are increasing in $x$ now follows from the proofs of Corollary 4.5 and Theorem 4.6 in [5].

We now address what we mean by a solution to (4.3) with nonlinearity (1.5). To solve (4.3), it is sufficient to regard $f_{1}\left(\varphi_{e}\right), f_{1}\left(\varphi_{o}\right),(1.5), h_{e}(x)$, and $h_{o}(x)$ as set valued functions, with $f$ and $h$ singleton sets for $\varphi_{e} \neq a$ and $\varphi_{o} \neq a$, and $f_{1}(a)=[a-1, a]$ and $h(0)=[0,1]$. By a solution to (4.3), we mean that $\varphi_{e}, \varphi_{o} \in C(\mathbb{R})$ and the differential inclusions

$$
\begin{aligned}
& -c_{e} \varphi_{e}^{\prime}(x) \in \alpha_{o}\left[\varphi_{o}(x)-\varphi_{e}(x)\right] \alpha_{e}\left[\varphi_{o}(x-2)-\varphi_{e}(x)\right]-\varphi_{e}(x)+h_{e}(x) \\
& -c_{o} \varphi_{o}^{\prime}(x) \in \alpha_{e}\left[\varphi_{e}(x+2)-\varphi_{o}(x)\right] \alpha_{o}\left[\varphi_{e}(x)-\varphi_{o}(x)\right]-\varphi_{o}(x)+h_{o}(x)
\end{aligned}
$$

hold for $x \in \mathbb{R}$.

4.2. The $a\left(c_{e}, c_{o}\right)$ relation. We now analyze the relationship between the wave speeds $c_{e}, c_{o}$ and the detuning parameter $a$.

For $c_{e}, c_{o} \neq 0$, let $r=r_{e}-r_{o}$ and set

$$
\begin{aligned}
& \Gamma\left(c_{e}, c_{o}\right)=a-\frac{1}{2} \\
& =\frac{c_{e}+c_{o}}{2 \pi} \int_{0}^{\infty} \frac{-A(s)+2\left[\alpha_{o}\{1+\cos s(1+r)\}+\alpha_{e}\{1+\cos s(1-r)\}+1\right]\left(\alpha_{e}+\alpha_{o}+1\right)}{A^{2}(s)+B^{2}(s)} d s,
\end{aligned}
$$

where

$$
\begin{aligned}
& A(s)=1-c_{e} c_{o} s^{2}+2 \alpha_{e} \alpha_{o}(1-\cos (2 s))+2\left(\alpha_{e}+\alpha_{o}\right), \\
& B(s)=\left(c_{e}+c_{o}\right) s\left(\alpha_{e}+\alpha_{o}+1\right) .
\end{aligned}
$$

Throughout this subsection, $c_{e}$ and $c_{o}$ are subject to the constraint

$$
F\left(\alpha_{e}, \alpha_{o}, c_{e}, c_{o}, r\right) \equiv \int_{0}^{\infty} \frac{\left[s\left(c_{e}-c_{o}\right)+2 \alpha_{o} \sin s(1+r)-2 \alpha_{e} \sin s(1-r)\right] A(s)}{s\left(A^{2}(s)+B^{2}(s)\right)} d s=0 .
$$

Observe that $F(\alpha, \alpha, c, c, 0)=0$ for $\alpha>0$ and $c \neq 0$. 
Let $c=\left(c_{e}+c_{o}\right) / 2$. With the simple change of variable $s \rightarrow s / c, \Gamma$ can be written as

$\Gamma(c) \equiv \Gamma\left(c_{e}, c_{o}\right)$

$$
=\frac{1}{\pi} \int_{0}^{\infty} \frac{-A(s / c)+2\left[\alpha_{o}\{1+\cos [(s / c)(1+r)]\}+\alpha_{e}\{1+\cos [(s / c)(1-r)]\}+1\right]\left(\alpha_{e}+\alpha_{o}+1\right)}{A^{2}(s / c)+B^{2}(s / c)} d s
$$

for $c_{e} \neq 0$ and $c_{o} \neq 0$.

THEOREM 4.1. Let $\varphi(\xi)$ be a solution to (1.9) with (4.1), (1.5), and (2.4), so that $\Gamma(c)$ is defined by (4.15) with $c_{e}, c_{o} \neq 0$. Then

$$
\lim _{c \rightarrow \infty}|\Gamma(c)| \leq \lim _{c_{e}, c_{o} \rightarrow \infty}\left|\Gamma\left(c_{e}, c_{o}\right)\right|=\frac{1}{2} .
$$

Proof. Let $c_{e}, c_{o} \neq 0$. Then

$$
\text { (4.19) } \lim _{c_{e}, c_{o} \rightarrow \infty}\left|\Gamma\left(c_{e}, c_{o}\right)\right|=\frac{1}{\pi} \int_{0}^{\infty} \frac{s^{2}+2 d+1}{s^{4}+2(d+1) s^{2}+2 d+1} d s=\frac{1}{\pi} \int_{0}^{\infty} \frac{1}{s^{2}+1} d s=\frac{1}{2},
$$

and if either $c_{e}$ or $c_{o}$ is finite

$$
\lim _{c \rightarrow \infty}|\Gamma(c)|=\frac{1}{\pi} \int_{0}^{\infty} \frac{2 d+1}{(2 d+4) s^{2}+2 d+1} d s<\frac{1}{\pi} \int_{0}^{\infty} \frac{1}{s^{2}+1} d s=\frac{1}{2},
$$

where $d=2\left(\alpha_{e}+\alpha_{o}\right)\left(\alpha_{e}+\alpha_{o}+1\right)$. If $c=c_{e}=c_{o}$, then $\lim _{c \rightarrow \infty}|\Gamma(c)|=1 / 2$ by (4.19).

5. Numerical results. In this section we focus on several numerical examples of the spatially varying diffusion coefficient traveling wave system

$$
\begin{aligned}
-c_{j}(t) & \phi^{\prime}\left(\xi_{j}\right)-\gamma \phi^{\prime \prime}\left(\xi_{j}\right) \\
& =\alpha_{j+1}\left[\phi\left(\xi_{j+1}+1\right)-\phi\left(\xi_{j}\right)\right]+\alpha_{j}\left[\phi\left(\xi_{j-1}-1\right)-\phi\left(\xi_{j}\right)\right]-f_{2}\left(\phi\left(\xi_{j}\right), a\right),
\end{aligned}
$$

where $j \in \mathbb{Z}$ and $f_{2}$ is given by (1.6). One of the primary difficulties in developing analytical results, including solutions, to differential-difference models is the unknown nature of the phenomena they contain. Numerically obtained results have the potential to point out the path to follow in our analytical investigations. The examples here were obtained using the algorithms presented in [8] and [9], numerical tools for investigating a large class of both linear and nonlinear traveling wave differential-difference equations (see also [1], [2], [3], [6], [7], [13]).

The following numerical examples are presented in two sections. In the first section we hold time fixed at $t=0$ and explore the $\left(\varphi_{k}(x), c_{k}\right)$ solutions. This section consists of three numerical examples. The first example is a presentation of the solution and $a(c)$ relation for a reaction-diffusion differential-difference equation with period two diffusion coefficient. In the second example we apply the period two diffusion formulation (4.1) through (4.3) to the constant diffusion problem. In this example we see period two bifurcation in the $a(c)$ relation and solution set. Our last example demonstrates the period four diffusion coefficient. The second section of numerical examples is dedicated to the time evolution on our nonlinear period two problem. In this section we explore the $u_{j}(t)$ solution plots. 
Although the equations in system (1.9) do not include the term $\gamma \varphi^{\prime \prime}\left(\xi_{j}\right)$, we include it in the equations of system (5.1) so that we may apply our numerical solvers. In each example, $\gamma$ is three orders of magnitude smaller than $\min \left(\alpha_{j}\right)$. We want to find the solution pairs $\left(\varphi_{k}(x, a), c_{j}(t, a)\right)$ to $(5.1)$ with boundary conditions $\varphi_{k}(-\infty, \cdot)=0$ and $\varphi_{k}(\infty, \cdot)=1, k=-N_{l}, \ldots, N_{r}$. But, since we are using a numerical solution method, we truncate the interval for $x$ to $[-T+1, T]$ and approximate the tails of the solution with asymptotic boundary conditions. This entails defining the boundary functions

$$
\varphi_{k}(x)=g_{k}^{l}(x) \quad \text { for } \quad x \leq-T+1 \quad \text { and } \quad \varphi_{k}(x)=g_{k}^{r}(x) \quad \text { for } \quad x \geq T
$$

for the delay terms, where the function $g_{k}^{l}(x) \in C^{1}$ asymptotically approaches zero as $x \rightarrow-\infty$ and the function $g_{k}^{r}(x) \in C^{1}$ asymptotically approaches one as $x \rightarrow \infty$, along with the boundary conditions

$$
\varphi_{k}^{\prime}(-T+1)=d g_{k}^{l}(-T+1) / d x \text { and } \varphi_{k}^{\prime}(T)=d g_{k}^{r}(T) / d x
$$

for the differential terms, $k=-N_{l}, \ldots, N_{r}$. In the numerical experiments presented here, we define

$$
g_{k}^{l}(x)=\kappa_{k}^{l} e^{b_{k}^{l} x} \text { and } g_{k}^{r}(x)=\left(1-\kappa_{k}^{r} e^{b_{k}^{r} x}\right) \text { for } b_{k}^{l}, b_{k}^{r}, \kappa_{k}^{l}, \kappa_{k}^{r} \in \mathbb{R}^{+}, k \in\left\{-N_{l}, \ldots, N_{r}\right\} .
$$

The numerical algorithms that we use to solve this problem are iterative solvers. Letting $\varphi_{k, p}$ represent the solutions at the previous iteration, we determine $b_{k}^{l}, b_{k}^{r}, \kappa_{k}^{l}$, and $\kappa_{k}^{r}$ for the current iteration by

$$
\begin{array}{r}
g_{k}^{l}(-T+2)=\varphi_{k, p}(-T+2), g_{k}^{r}(T-1)=\varphi_{k, p}(T-1), \\
d g_{k}^{l}(-T+2) / d x=\varphi_{k, p}^{\prime}(-T+2), \text { and } d g_{k}^{r}(T-1) / d x=\varphi_{k, p}^{\prime}(T-1) .
\end{array}
$$

6. Time $\boldsymbol{t}=\mathbf{0}$. In the analytic sections of this paper we fixed time and solved. In this section we consider numerical examples where we solve for $\varphi_{k}$ for time fixed at $t=0$. Recall that this implies $r_{k}=\int_{0}^{0} c_{k}(s) d s=0, k=-N_{l}, \ldots, N_{r}$.

6.1. Example 1: Period two diffusion coefficient. Typically the period two diffusion coefficient problem will consist of two solution curves, $\varphi_{e}$ and $\varphi_{o}$, each with its own corresponding wave speed. This indicates that we have two possible trajectories that points in the lattice follow from one phase to the other. Since $\alpha$ is period two on the lattice, every other lattice point follows the same trajectory.

In terms of the two solution curves, with $t=0, \varphi_{e}(x)=\phi(x)$ and $\varphi_{o}(x)=\phi(x+1)$, (4.3), the boundary requirements (5.2) and (5.3) are

$$
\varphi_{o}(x)=g_{o}^{l}(x+1) \quad \text { for } \quad x \leq-T, \quad \varphi_{e}(x)=g_{e}^{r}(x) \quad \text { for } \quad x \geq T,
$$

and

$$
\begin{array}{r}
\varphi_{o}^{\prime}(-T)=d g_{o}^{l}(-T+1) / d x, \varphi_{o}^{\prime}(T)=d g_{o}^{r}(T+1) / d x \\
\varphi_{e}^{\prime}(T)=d g_{e}^{r}(T) / d x, \varphi_{e}^{\prime}(-T)=d g_{e}^{l}(-T) / d x
\end{array}
$$

In this example we are solving, on the truncated domain, (5.1) with $\gamma=10^{-3}$, the nonlinearity coefficient $d=15$, and $\alpha_{j}$ varying with period two. We represent this problem as the system of two equations

$$
\begin{aligned}
& -c_{e} \varphi_{e}^{\prime}(x)-\gamma \varphi_{e}^{\prime \prime}(x)=\alpha_{o}\left[\varphi_{o}(\xi)-\varphi_{e}(x)\right]+\alpha_{e}\left[\varphi_{o}(x-2)-\varphi_{e}(x)\right]-f_{2}\left(\varphi_{e}(x), a\right), \\
& -c_{o} \varphi_{o}^{\prime}(x)-\gamma \varphi_{o}^{\prime \prime}(x)=\alpha_{e}\left[\varphi_{e}(x+2)-\varphi_{o}(x)\right]+\alpha_{o}\left[\varphi_{e}(x)-\varphi_{o}(x)\right]-f_{2}\left(\varphi_{o}(x), a\right)
\end{aligned}
$$




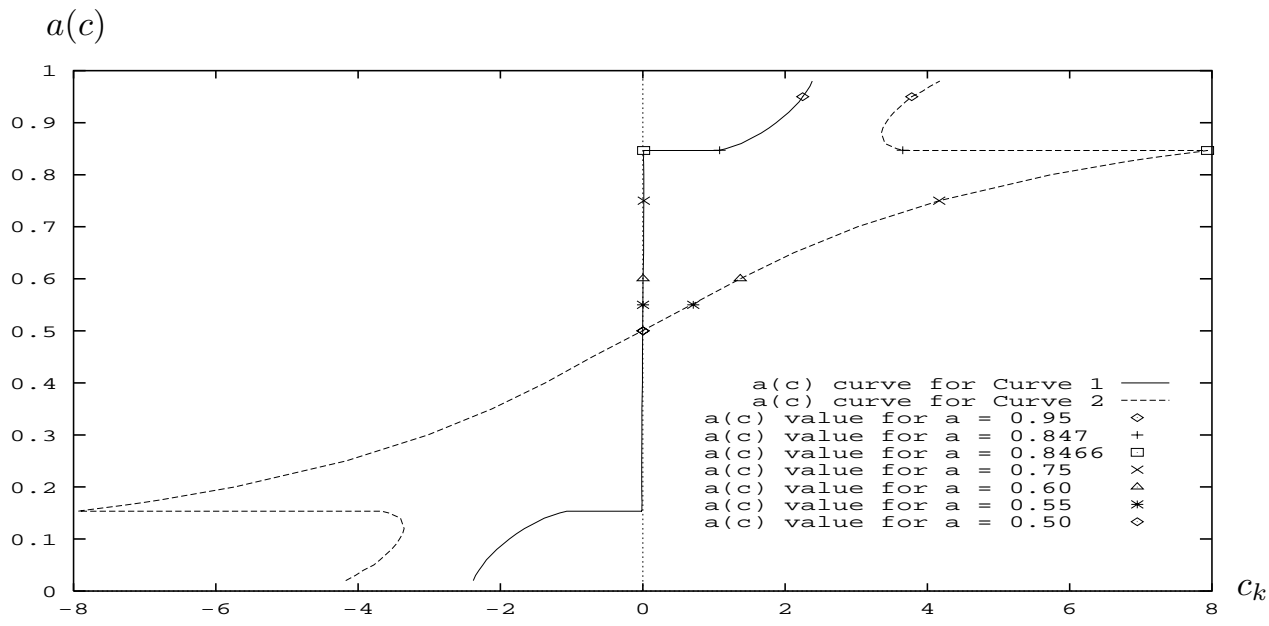

FIG. 1. Example 1: An a(c) plot for the period two pair of diffusion coefficients $\alpha=1.3,1.9$.

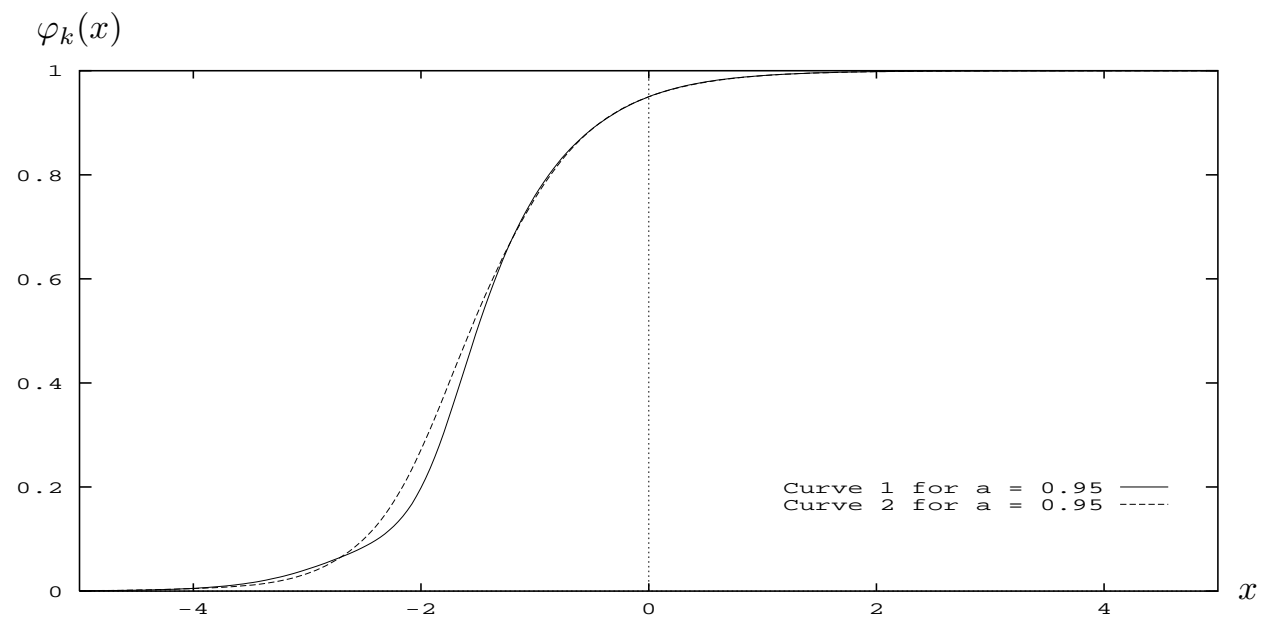

FIG. 2. Example 1: A solution plot for the period two pair of diffusion coefficients $\alpha=1.3,1.9$.

with the above boundary requirements. In Figure 1 we present the $a(c)$ curve associated with this problem with the diffusion coefficients $\alpha_{e}=1.3$ and $\alpha_{o}=1.9$.

Remark 6.1.

(i) For each value of $a \neq 1 / 2$, there exist two different values of $c$, both of which are the same sign. This is in agreement with the analysis for this problem with $\gamma=0$ and nonlinearity $f_{1}$.

(ii) There exists a "jump" in the $c$ values for some $a \in(0.8466,0.8470)$ and for some $a \in(0.1530,0.1534)$. When $a \in(0.1534,0.8466)$ we see that one of the $a(c)$ curves, curve $\mathrm{C} 1$, has features (propagation failure) that we have come to expect for spatially discrete problems. The other $a(c)$ curve, curve $\mathrm{C} 2$, appears very much like an $a(c)$ relation for the spatially continuous version of our model.

(iii) One of the pair of $a(c)$ curves has a large interval for $a$ in which $c$ is small, $|c|<10^{-5}$, while the other curve of the pair has no such nontrivial interval. 


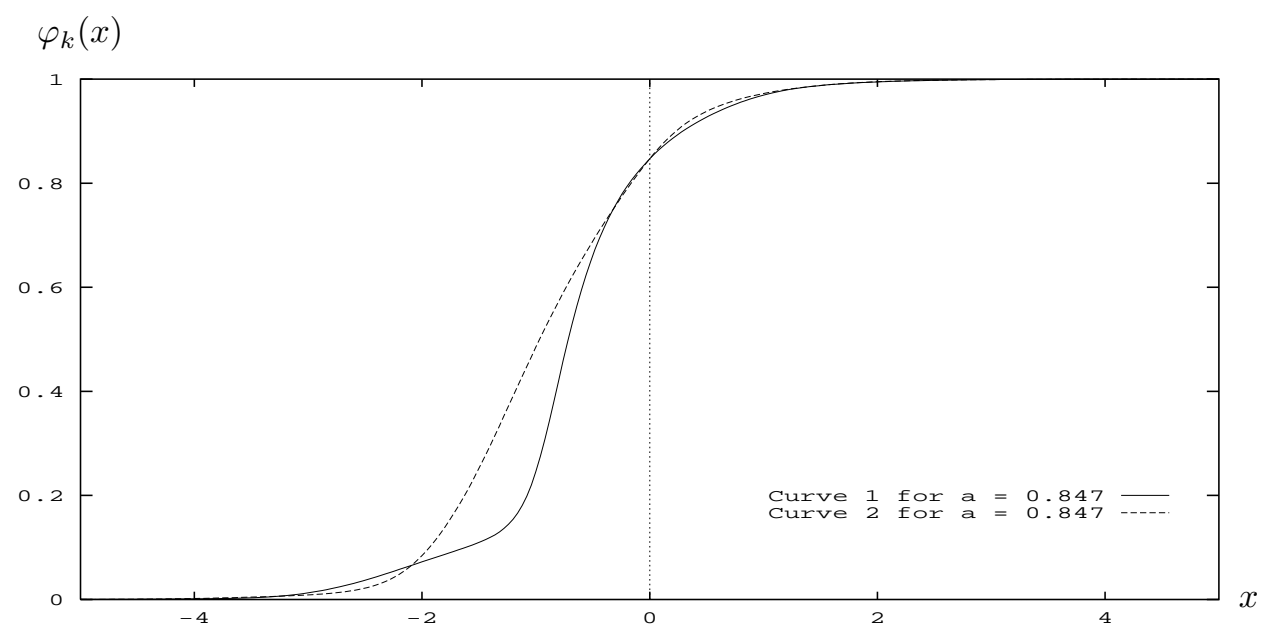

FIG. 3. Example 1: A solution plot for the period two pair of diffusion coefficients $\alpha=1.3,1.9$.

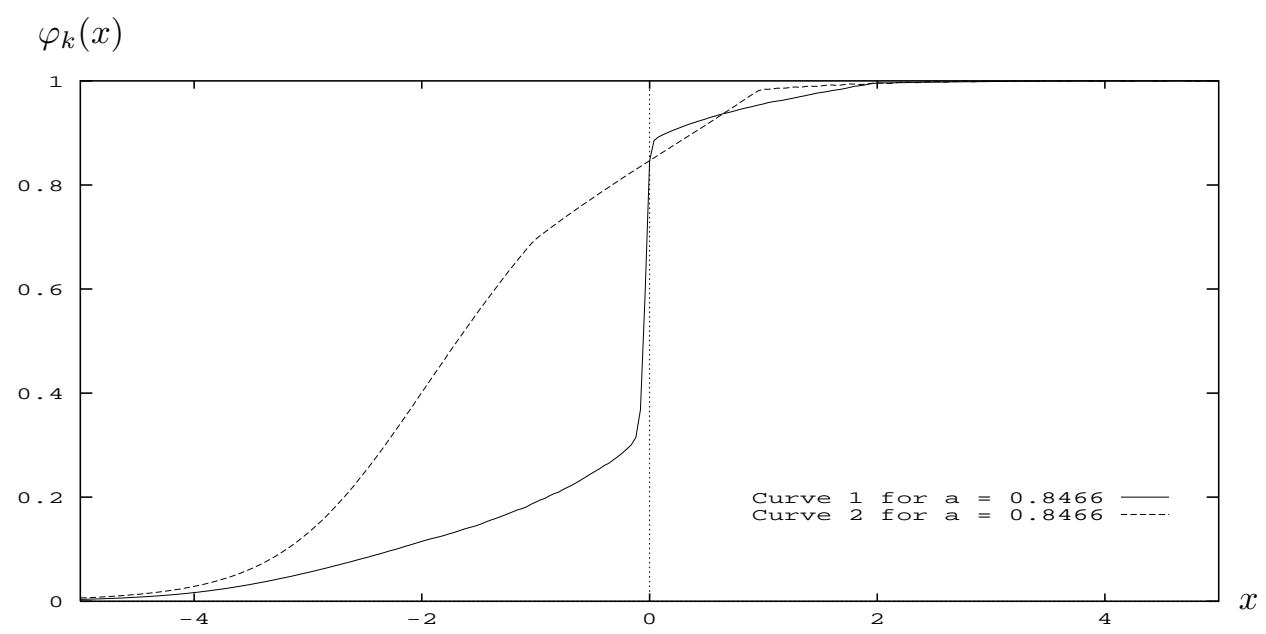

Fig. 4. Example 1: A solution plot for the period two pair of diffusion coefficients $\alpha=1.3,1.9$.

This implies the possibility of propagation failure for every other point in the lattice for a nontrivial interval of the detuning parameter $a$.

Figures 2-8 are the corresponding solution plots for selected values of $a$. Recall that for this problem the chosen phase conditions are $\varphi_{e}(0)=a$ and $\varphi_{o}(-1)=a$. Each solution plot consists of two curves, the functions $\varphi_{e}(\xi)$ and $\varphi_{o}(\xi+1)$. We shift the function $\varphi_{o}$ when plotting to make comparison of the two solution profiles easier. Starting with $a=0.95$, Figure 2, we show how the pairs of solution curves associated with the $a(c)$ values progress as $a$ decreases to 0.5 , Figure 8 .

Remark 6.2. There exists a jump in the progression of the solution curves as $a$ varies from $a=0.8470$ to $a=0.8466$ (Figures 3 and 4) corresponding to the jump in the wave speeds (Figure 1).

The solution pairs in Figures 2 and 3, where $a \geq 0.8470$, change curvature only once, i.e., $\varphi_{e}^{\prime \prime}$ and $\varphi_{o}^{\prime \prime}$ equal zero for only one value of $\xi$. When $a \leq 0.8466$, Figures $4-8$, this is no longer true. The multiple changes in curvature is a property that the 


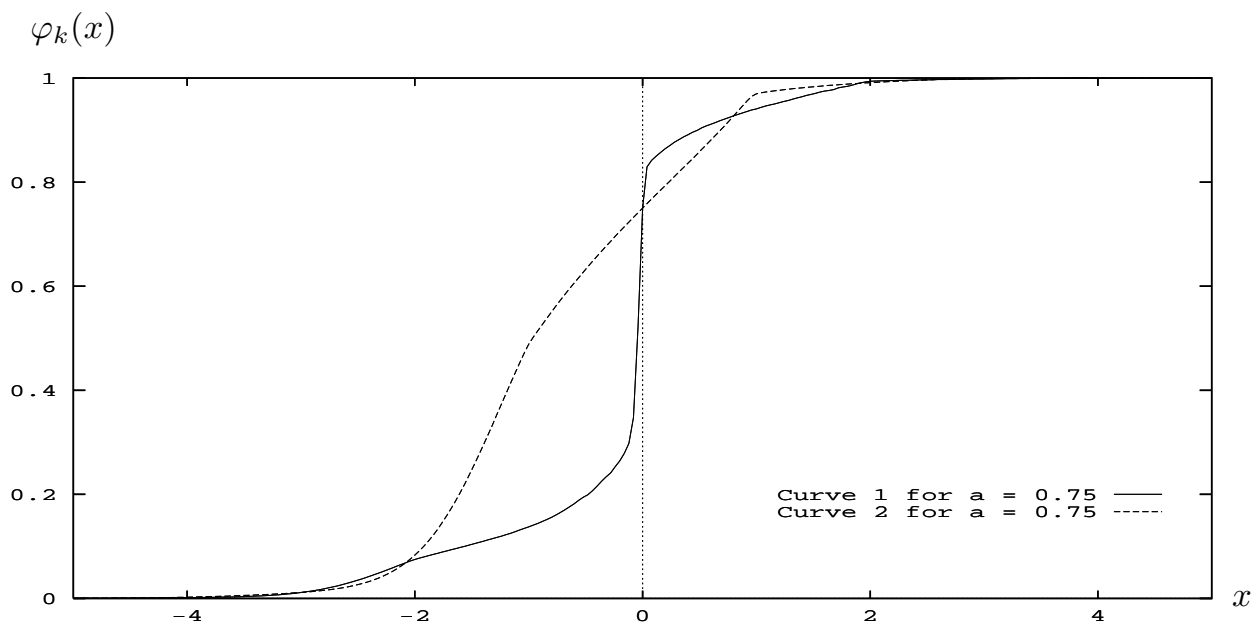

Fig. 5. Example 1: A solution plot for the period two pair of diffusion coefficients $\alpha=1.3,1.9$.

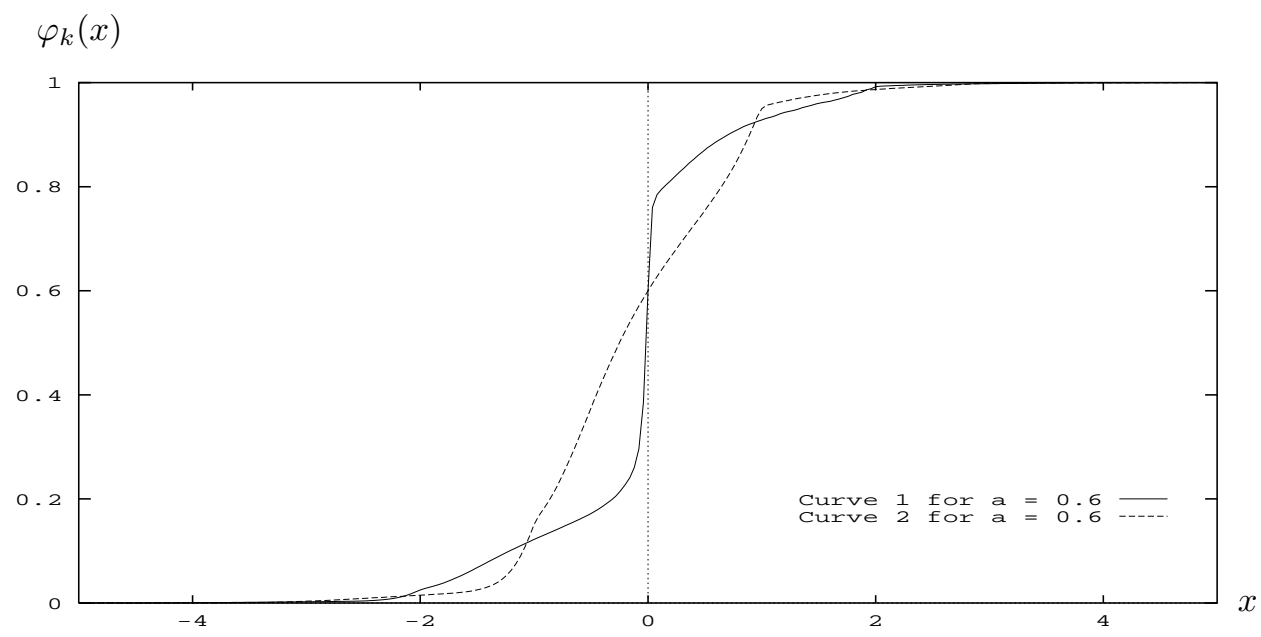

FIG. 6. Example 1: A solution plot for the period two pair of diffusion coefficients $\alpha=1.3,1.9$.

spatially continuous version of our reaction-diffusion models do not exhibit. These curves also reaffirm the fact that for values of $a$ for which we would expect large wave speeds, spatially continuous and spatially discrete models are more similar than for small wave speeds.

6.2. Example 2: Constant diffusion, variable wave speed, and period two bifurcation. Suppose we set $\alpha_{j}$ to some positive fixed value for all $j \in \mathbb{Z}$ but we still treat the problem as if $\alpha_{j}$ varied with period two. Then (5.1) becomes

$$
\begin{aligned}
& -c_{e} \varphi_{e}^{\prime}(x)-\gamma \varphi_{e}^{\prime \prime}(x)=\alpha\left[\varphi_{o}(x)-2 \varphi_{e}(x)+\varphi_{o}(x-2)\right]-f_{2}\left(\varphi_{e}(x), a\right), \\
& -c_{o} \varphi_{o}^{\prime}(x)-\gamma \varphi_{o}^{\prime \prime}(x)=\alpha\left[\varphi_{e}(x+2)-2 \varphi_{o}(x)+\varphi_{e}(x)\right]-f_{2}\left(\varphi_{o}(x), a\right),
\end{aligned}
$$

a constant coefficient problem where the wave speed is allowed to vary with period two. If we set $\varphi_{e}(x+1)=\varphi_{o}(x)$ and $c_{e}=c_{o}$, then we have the constant coefficient 


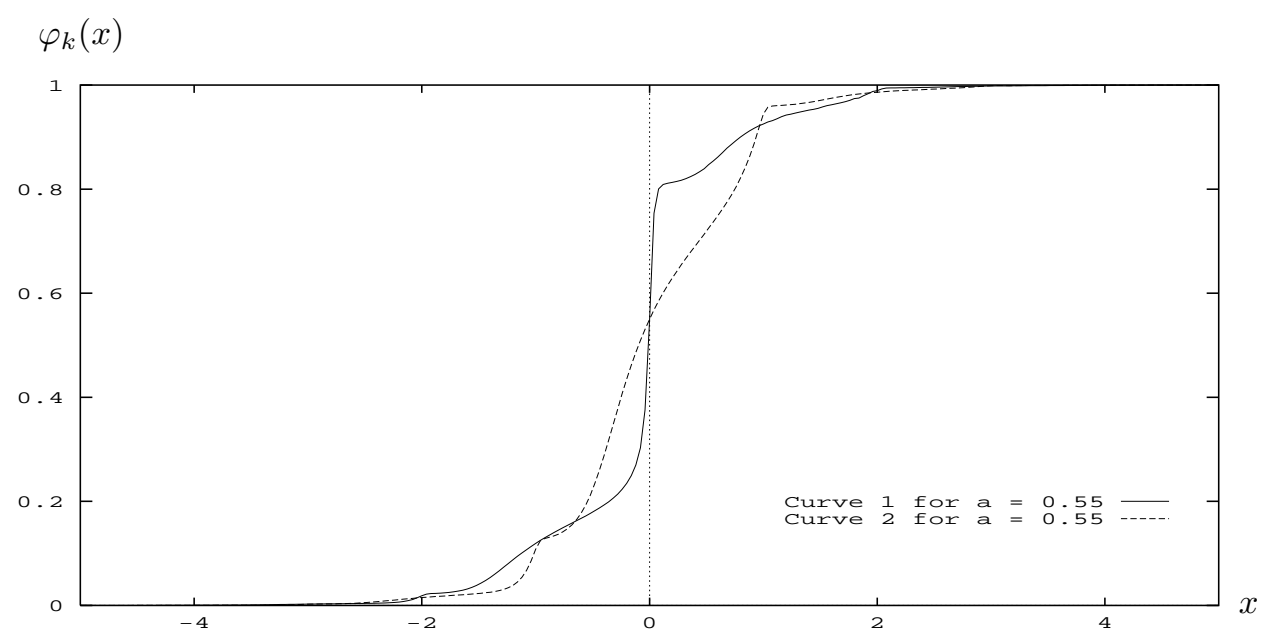

Fig. 7. Example 1: A solution plot for the period two pair of diffusion coefficients $\alpha=1.3,1.9$.

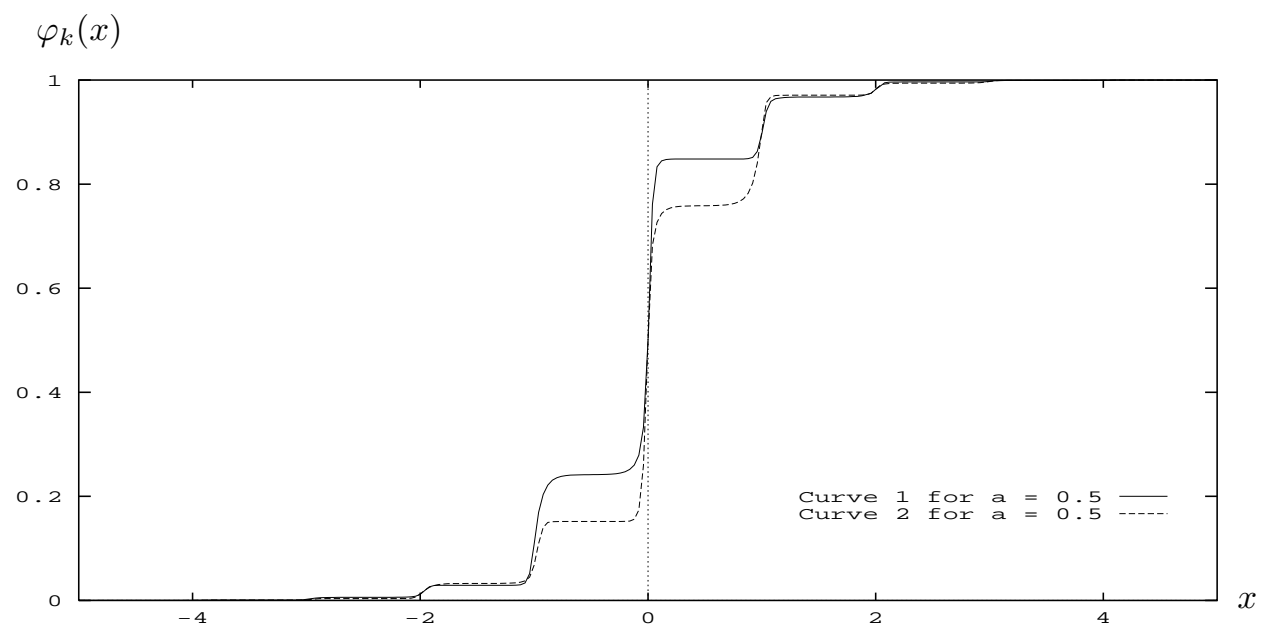

FIG. 8. Example 1: A solution plot for the period two pair of diffusion coefficients $\alpha=1.3,1.9$.

equation we solved for in [8]. In this example $\gamma=10^{-3}, d=15$, and $\alpha=1$.6. We begin with Figure 9, where we have plotted the $a(c)$ curve for the period two wave speed case (the curves labeled $\mathrm{c} 2$ ), along with the $a(c)$ curve (the curve labeled c1) for the case where $c$ is assumed constant. The two $a(c)$ curves (c1 and $\mathrm{c} 2$ ) coincide except for two regions, one for $c>0$ and one for $c<0$.

Remark 6.3. The regions where the $a(c)$ curves do not coincide are bounded by four bifurcation points, two for each region. These are the points where the $a(c)$ curves split. Letting $\varphi=\left(\varphi_{e}, \varphi_{o}\right)$ and $c=\left(c_{e}, c_{o}\right)$, these points indicate the values of $a$ for which the fixed point of $(5.1),(\varphi, c)$, bifurcates. We will refer to these regions where the $a(c)$ curves $\mathrm{c} 1$ and $\mathrm{c} 2$ are distinct as the bifurcation regions or the regions of period two bifurcation.

Figures 10 and 13 are plots of solutions of the period two wave speed problem, outside the bifurcation regions. Figure 10 contains two pairs of solutions and Figure 13 contains three pairs of solutions. The solution pairs in these two plots correspond 


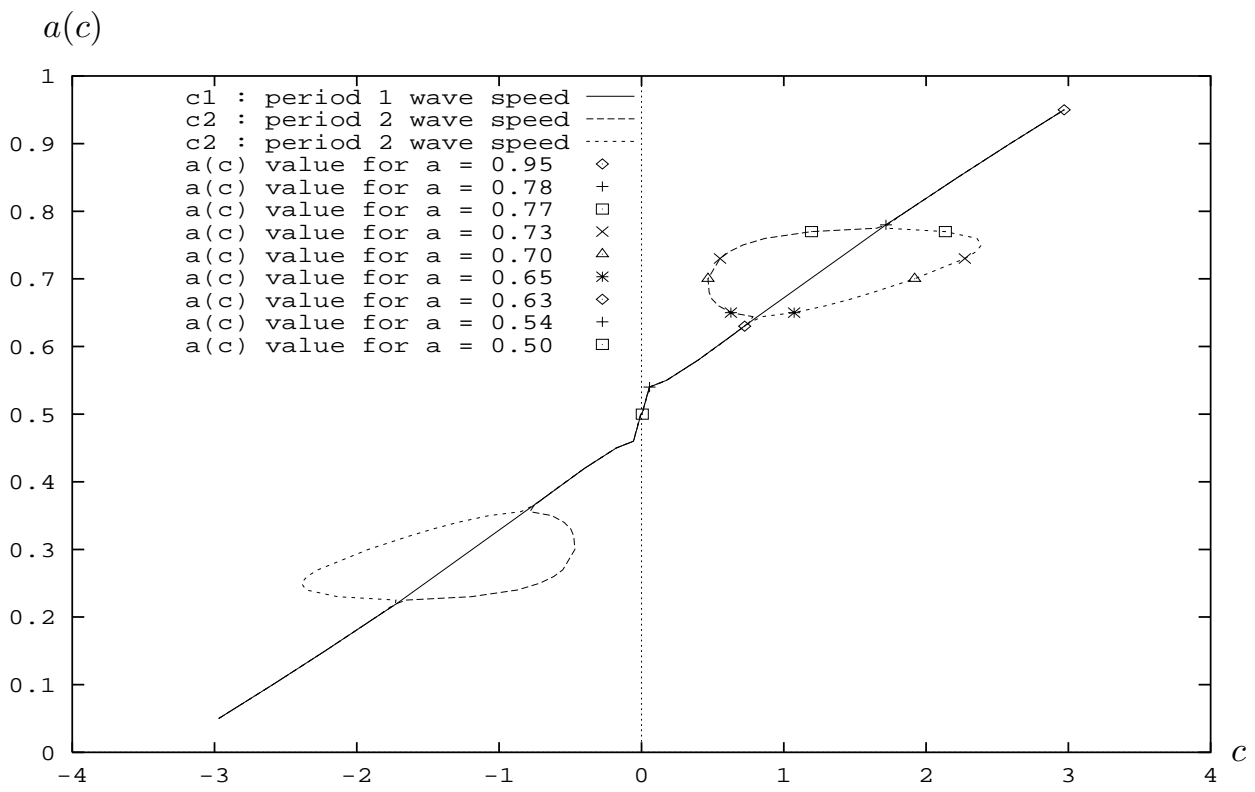

FIG. 9. Example 2: An a(c) plot for a constant coefficient problem.

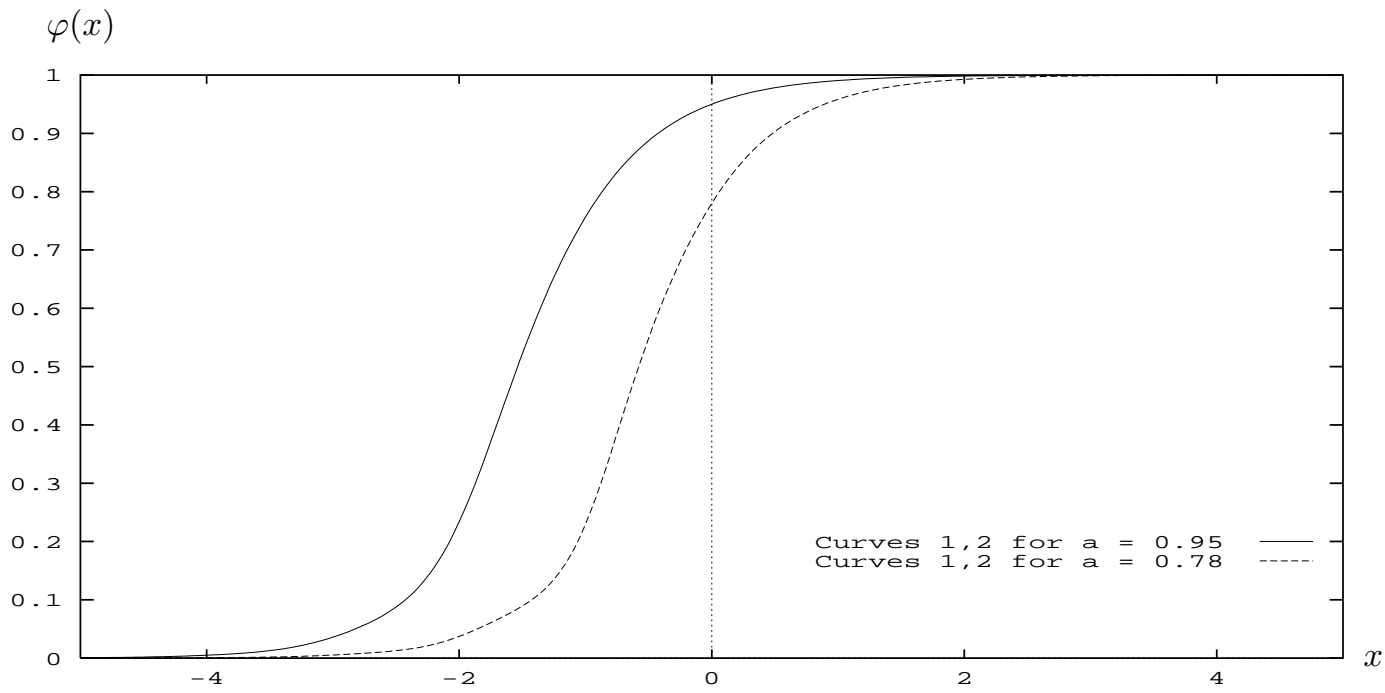

FIG. 10. Example 2: Constant diffusion coefficients. No period splitting.

to values of $a$ where $c_{e}=c_{o}$. Figures 10 and 13 show us that for these values of $a$, $\varphi_{e}(x+1)=\varphi_{o}(x)$ and $c_{e}=c_{o}$, that the shape of the waves are identical.

As we decrease $a$ from $a=0.78$ into the region of bifurcation, $(\varphi, c)$ goes through a period two bifurcation and we begin to obtain a pair of distinct solution trajectories and wave speeds for each $a$ (Figure 11, $a=0.77$ and $a=0.73$ ), one solution and wave speed similar to the solution and wave speed for $a=0.78$ (curve 2) and the other solution (curve 1) and wave speed moving towards the solution and wave speed for $a=0.63$. 


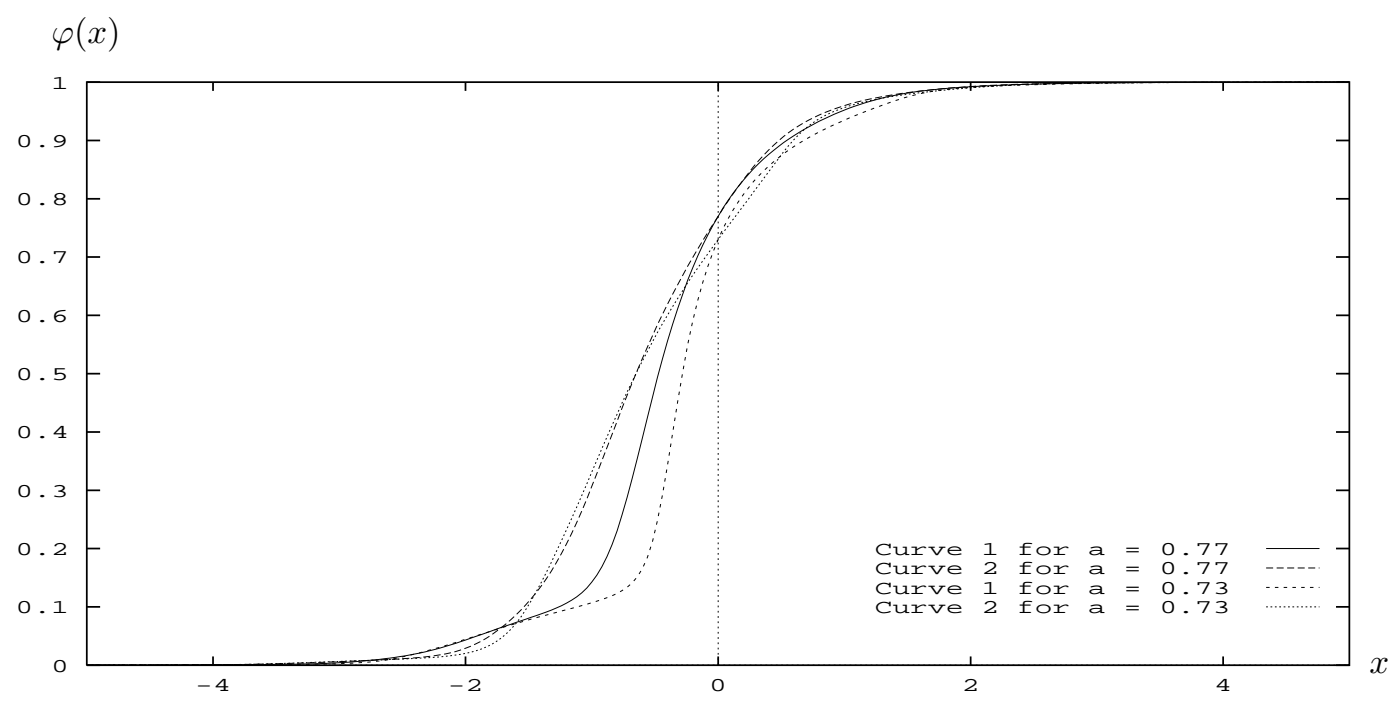

FIG. 11. Example 2: Constant diffusion coefficients. Period splitting.

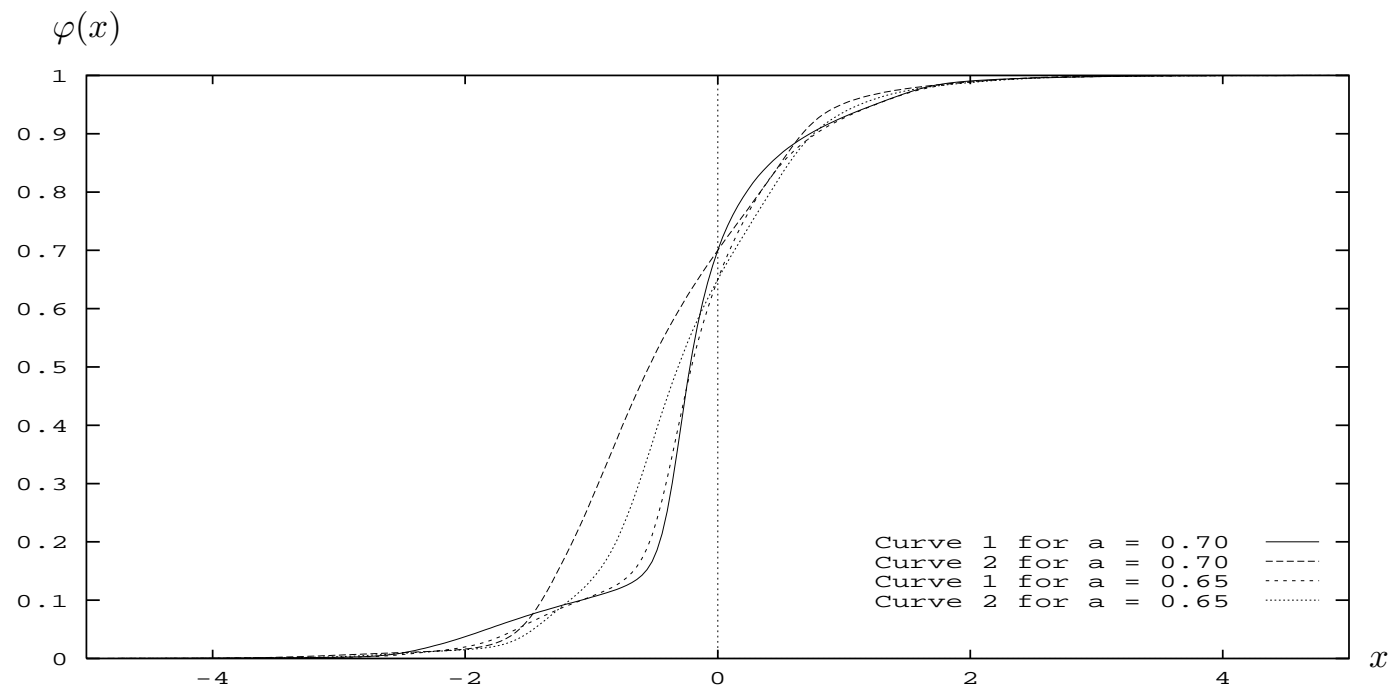

FIG. 12. Example 2: Constant diffusion coefficients. Period splitting.

Continuing to decrease $a$, once the solution represented by curve 1 has reached a neighborhood of the solution for $a=0.63$, curve 2 begins to approach this solution as well (Figure 12, $a=0.70$ and $a=0.65$ ).

As $a$ decreases further towards $a=0.63,(\varphi, c)$ goes through another period two bifurcation, and we once again have only one solution trajectory and one wave speed (Figure 13).

In the previous example (Example 1) we point out that we could classify the solution curves into two types, one where the solution curves change curvature only once and one where the solution curves change curvature more than once. In this example we can carry that classification one step farther. For $a \geq 0.78, \varphi_{e}$ and $\varphi_{o}$ change curvature only once. As $a$ crosses the bifurcation point near 0.78 into the region 


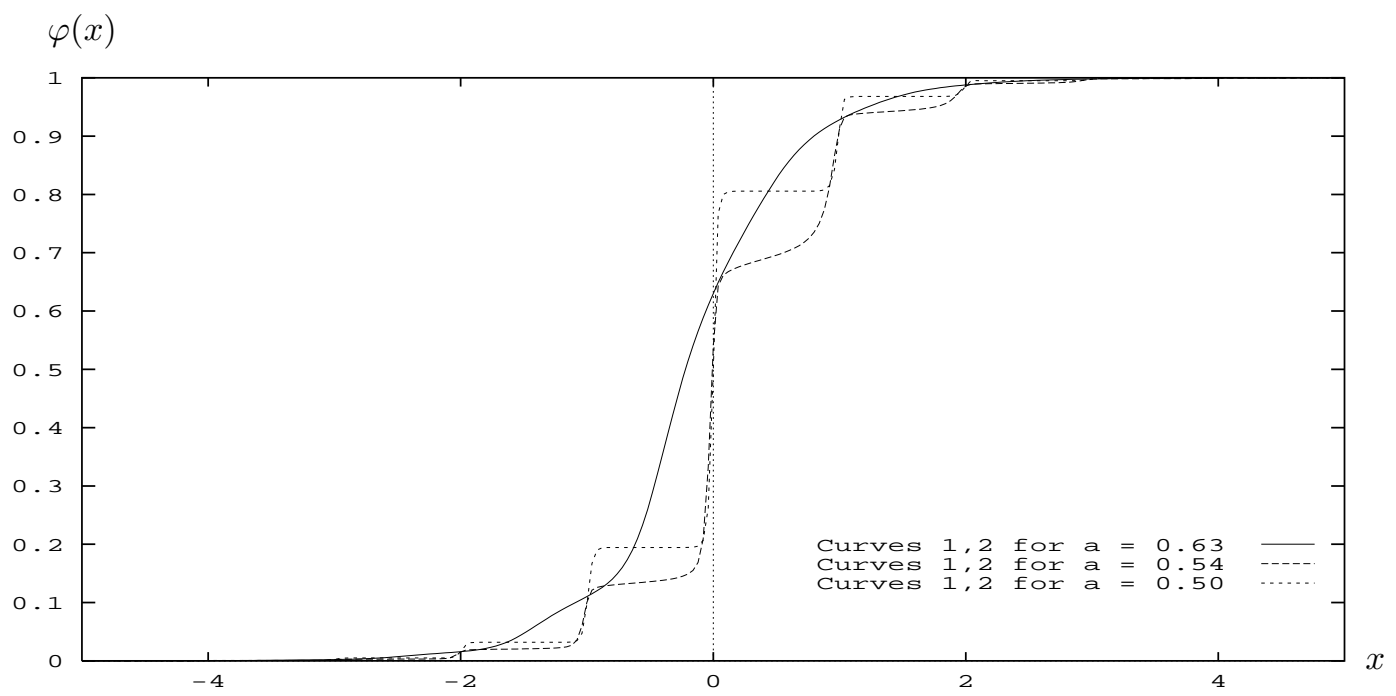

FIG. 13. Example 2: Constant diffusion coefficients. No period splitting.

of bifurcation, one of the solution curves gains additional curvature changes while the other curve of the pair has only one change in curvature. This is true throughout the bifurcation region. As a decreases from the region of bifurcation towards 0.50 and crosses the bifurcation point, both solution curves in the pair have more than one change in curvature. Through these plots, we can see that the bifurcation points separate the solutions type into distinct types.

6.3. Collection of $\boldsymbol{a}(\boldsymbol{c})$ curves. Figure 14 consists of a set of various $a(c)$ curve pairs of (5.1) with $\gamma=10^{-3}$ and $d=15$ for several sets of period two diffusion coefficients. The average of each pair of diffusion coefficients is 1.6.

Remark 6.4.

(i) Starting with the $a(c)$ curves for $\alpha=(1.6,1.6)$, we see in Figure 14 that as the distance between the two diffusion coefficient values increases, so does the distance between the two wave speeds.

(ii) There appears to be a vertical boundary, at $c=0$, and two horizontal boundaries, at $a \approx 0.1532$ and $a \approx 0.8468$, which restrict the $a(c)$ curve pairs. This may imply a restriction on the magnitude of the difference in the two diffusion coefficient values.

The $a(c)$ plots of Figure 14 were found by applying our Newton technique to finding the solution pair $(\varphi, c)$ for various values of $a$.

6.4. Example 3: Period four diffusion coefficient. This last example illustrates the solution set to (5.1) with $\gamma=2 \times 10^{-3}, d=15$, and $\alpha_{j}$ varies with period four over the values $1.4,1.5,1.6$, and 1.7. In this example we do not shift the solution plots when plotting. In Figures 15-18, each figure contains one set of solution curves. Table 1 contains the corresponding wave speeds. All four curves in Figures 15-17 contain multiple changes in curvature. The four curves in Figure 18 contain only one change in curvature. We have not included any figures for $a$ in the range where the set of curves would include both multiple and single changes of curvature because of the lack of stability of the iterative method applied to the system for such $a$ 's. 
$a(c)$

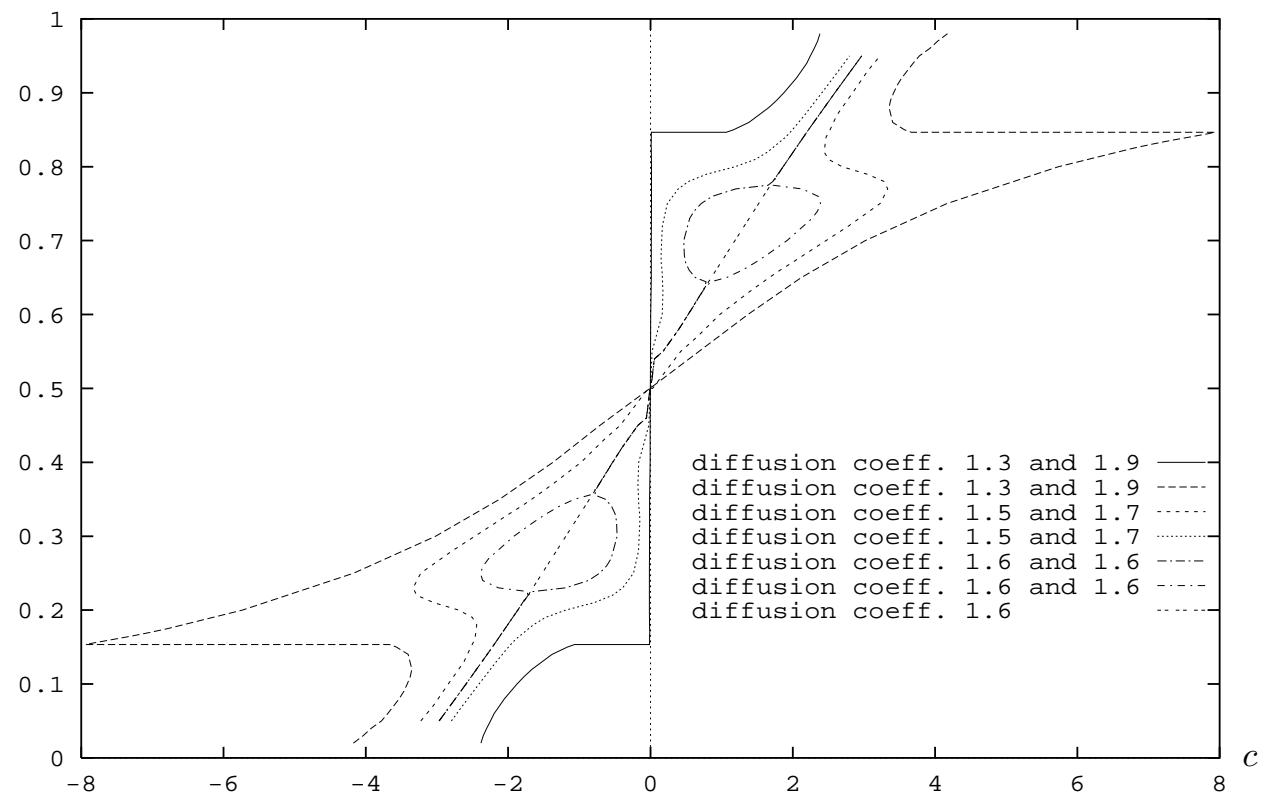

FIG. 14. A plot of a (c) curves for various choices of period 2 diffusion coefficients. The average of each pair of coefficients is 1.6. The a(c) curves appear in pairs.

TABLE 1

Wave speed c values for Figures 15, 16, 17, and 18.

\begin{tabular}{|c||c|c|c|c|}
\hline Figure 15 $a=0.50$ & Curve C1 & Curve C2 & Curve C3 & Curve C4 \\
\hline Wave Speed $c$ & $10^{-4}$ & $10^{-4}$ & $10^{-4}$ & $10^{-4}$ \\
\hline \hline Figure 16 $a=0.55$ & Curve C1 & Curve C2 & Curve C3 & Curve C4 \\
\hline Wave Speed $c$ & $3.70167 \times 10^{-2}$ & $5.01973 \times 10^{-2}$ & $1.26761 \times 10^{-1}$ & $4.39932 \times 10^{-1}$ \\
\hline \hline Figure 17 $a=0.60$ & Curve C1 & Curve C2 & Curve C3 & Curve C4 \\
\hline Wave Speed $c$ & $2.34119 \times 10^{-1}$ & $5.98346 \times 10^{-1}$ & $3.92691 \times 10^{-1}$ & $8.40303 \times 10^{-1}$ \\
\hline \hline Figure 18 $a=0.90$ & Curve C1 & Curve C2 & Curve C3 & Curve C4 \\
\hline Wave Speed $c$ & 2.06623 & 2.62750 & 2.75114 & 2.76231 \\
\hline
\end{tabular}

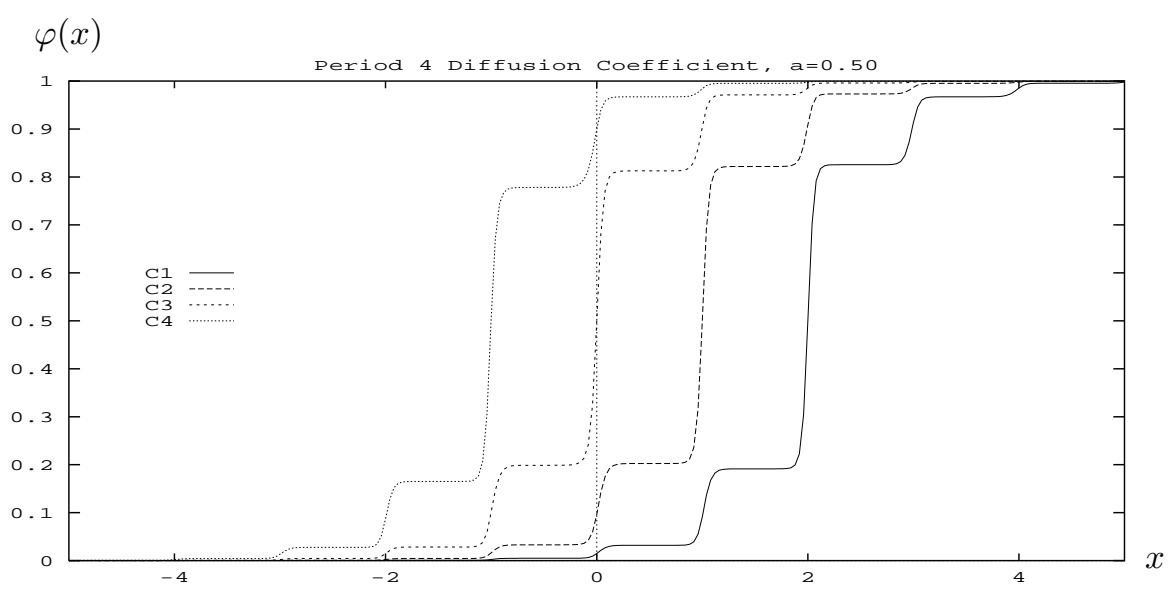

FiG. 15. Example 3: A solution plot for the period four diffusion coefficients $\alpha=1.4,1.5,1.6,1.7$. 


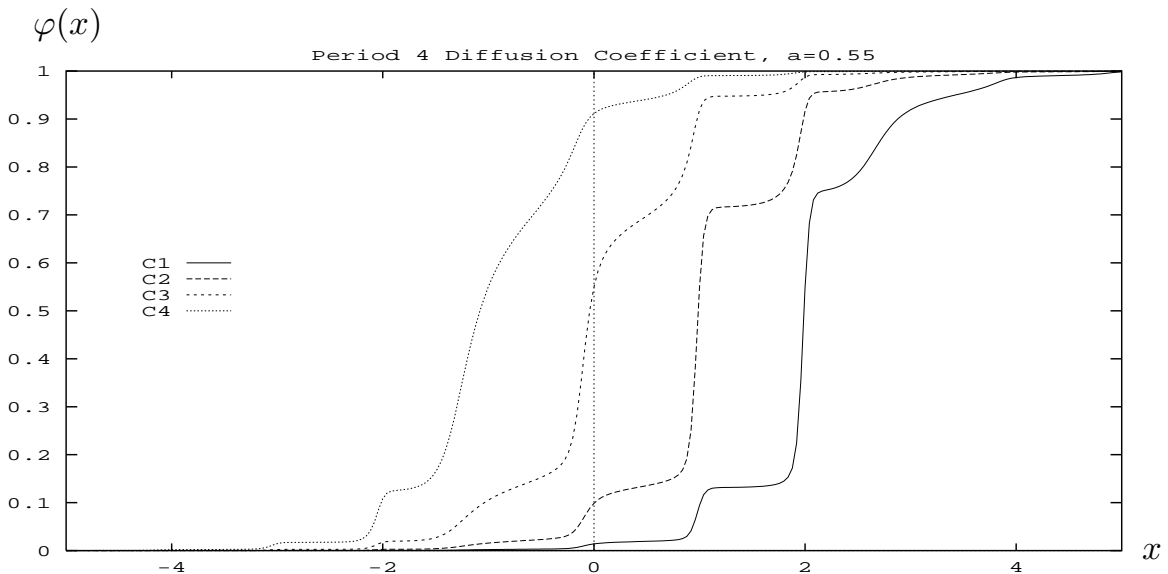

FIG. 16. Example 3: A solution plot for the period four diffusion coefficients $\alpha=1.4,1.5,1.6,1.7$.

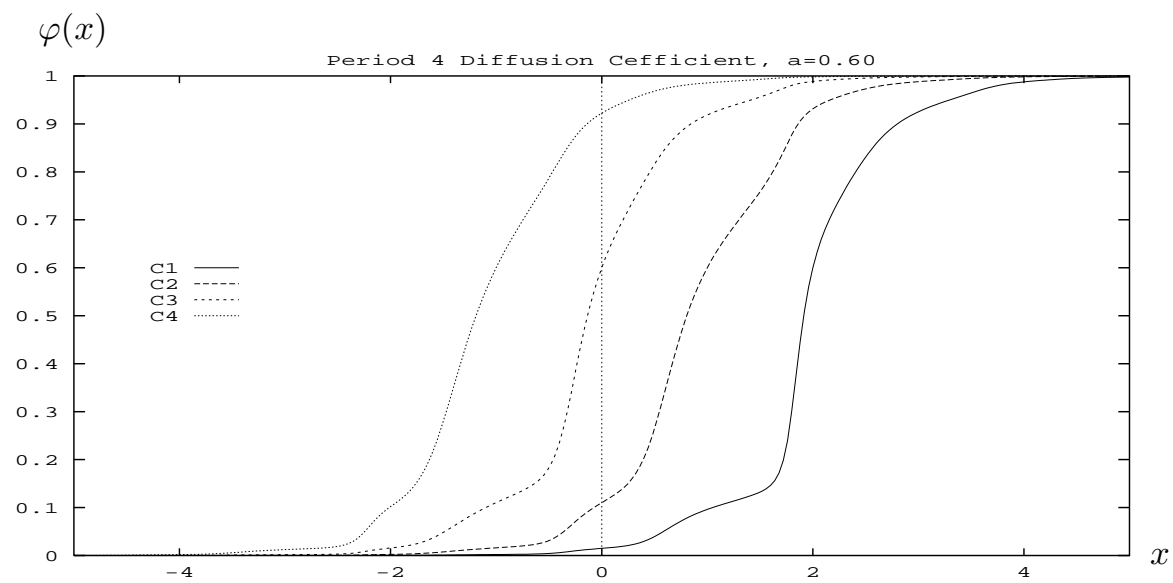

FIG. 17. Example 3: A solution plot for the period four diffusion coefficients $\alpha=1.4,1.5,1.6,1.7$.

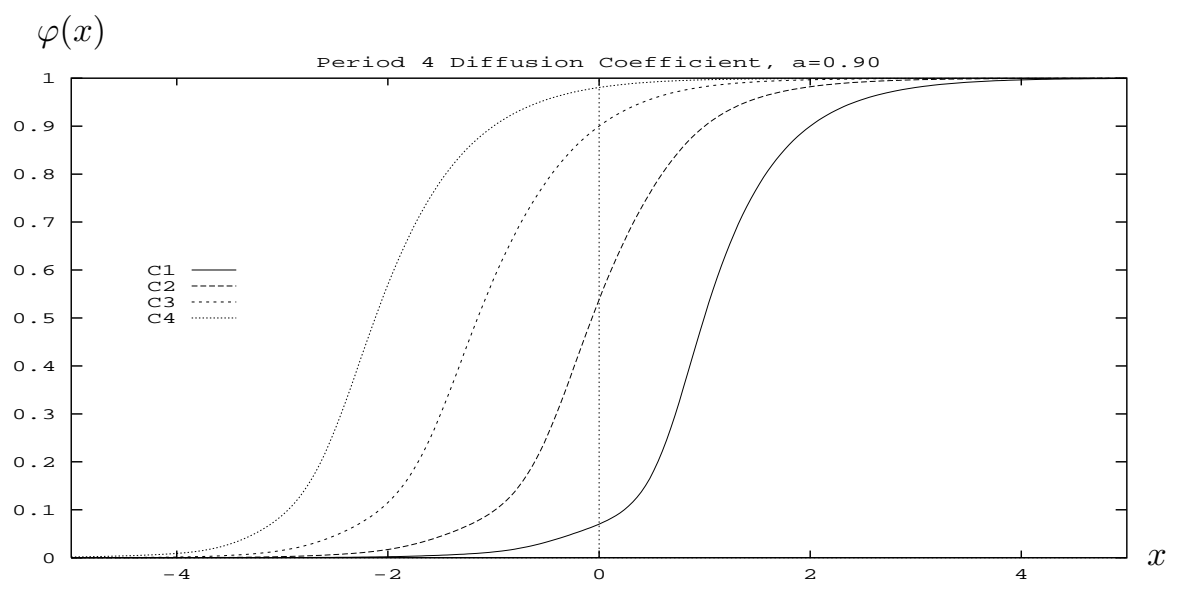

FIG. 18. Example 3: A solution plot for the period four diffusion coefficients $\alpha=1.4,1.5,1.6,1.7$. 
7. $\boldsymbol{u}_{\boldsymbol{j}}$ varies in $\boldsymbol{t}$. In this section we reconstruct the solution $u_{j}(t)$ and study the dependence of the wave forms and speeds on time. We specifically investigate the period two system (6.2) with diffusion values $\alpha_{e}=1.5$ and $\alpha_{o}=1.7$. The $a$ versus $c_{e}, c_{o}$ plot for this problem when $t=0$ and $r=0$ appears in Figure 14.

7.1. $c(r)$ maps. We begin our study of the time evolution of $u_{j}$ with a set of phase portraits. Recall $r_{e}=\int_{0}^{t} c_{e}(s) d s, r_{o}=\int_{0}^{t} c_{o}(s) d s, c_{e}=c_{e}(t)$, and $c_{o}=c_{o}(t)$, where $c_{e}=d r_{e} / d t$ and $c_{o}=d r_{o} / d t$. Let $c=c_{e}-c_{o}$ be the difference in wave speeds. Recalling the phase conditions $\varphi_{e}\left(r_{e}\right)=a$ and $\varphi_{o}\left(-1+r_{o}\right)=a$, the quantity $r=r_{e}-r_{o}$ is a measure of the time dependent phase shift between $\varphi_{e}$ and $\varphi_{o}$. This means that $c=d r / d t$, i.e., that the time dependent difference in wave speed is the derivative of the phase shift.

Figures 19(i)-19(v) are phase portraits, $c$ versus $r$, for various $a$. Figure 19(i) shows us, that for $a=0.6,0.625$, the difference in wave speeds $c \rightarrow 0$ as $t$ goes from zero to $\infty$. The same is true for $a=0.775$ and $a=0.8$, Figure 19(v). As time increases from zero, the phase shift increases until the wave speeds match. The values of $a$ represented in Figures 19(i) and 19(v) have solution pairs $\varphi_{e}, \varphi_{o}$ which possess either both one change in curvature, Figure 19(v), or both more than one change in curvature, Figure 19(i).

In Figures 19(ii), 19(iii), and 19(iv), for $a=0.65,0.7$, and 0.75, respectively, we see something quite different. In each case the phase map has an hysteresis loop. As time increases from zero, instead of $c \rightarrow 0$, the value of $c$ and the shift $r$ get caught in a loop and will continue to travel around that loop as $t \rightarrow \infty$. The figures with the hysteresis loops represent values of $a$ for which one of the solution pair $\varphi_{e}, \varphi_{o}$ changes curvature once while the other solution changes curvature more than once. The jumps in the loop are formed when the value of $|c|$ decreases and the two solutions switch their curvature type.

As we have seen in Figure 9, there exist four values of $a$, two less that 1/2, two greater that $1 / 2$, at which there is a bifurcation in the behavior of $c_{e}, c_{o}$ and $\varphi_{e}, \varphi_{o}$. Here we have quantified it in terms of whether the $c(r)$ curve has a hysteresis loop or not. The value $a=0.775$ is close to a bifurcation point; note the extremely large slope in its $c(r)$ curve, Figure 19(v).

7.2. $\boldsymbol{u}_{\boldsymbol{j}}(\boldsymbol{t})$ plots. We now present the solution plots for system (6.2) with $\alpha_{e}=$ 1.7 and $\alpha_{o}=1.5$ along with the $c_{e}(t), c_{o}(t)$ curves. Figure 20 shows $r_{e}$ versus $r_{o}$ for $a=0.6,0.7$, and 0.8. For $a=0.6$ and $0.8, r_{e}$ and $r_{o}$ become linearly related as $t \rightarrow \infty$.

The solution plots for $a=0.6$ appear in Figures 21 and 22 and the $c_{e}, c_{o}$ plot appears in Figure 23. In Figure 21 we plotted the value of $u_{j}$ at the transition zone for several values of time $t$ allowing us to observe how the wave flows along the lattice. The time trajectories for several $u_{j}$ appear in Figure 22. The points on this figure correspond with the points of Figure 21. In Figure 23 we see that although the values of $c_{e}(0) \neq c_{o}(0), c_{e}(t)$ and $c_{o}(t)$ approach each other asymptotically in time. These three plots for $a=0.6$ are representative of the solutions, $\left(\varphi_{e}, c_{e}\right)$ and $\left(\varphi_{o}, c_{o}\right)$, which possess multiple changes of curvature, where the values of $a$ are nearest $1 / 2$.

Solution plots for $a=0.8$ appear in Figures 24 and 25 and the $c_{e}, c_{o}$ plot appears in Figure 26. In Figure 26 we see that although the values of $c_{e}(0) \neq c_{o}(0), c_{e}(t)$ and $c_{o}(t)$ approach each other asymptotically in time. In the time trajectory plot, Figure 25 , we see how the flow of the lattice points from one phase to the other changes (even reversing directions) as $c_{e}(t)$ and $c_{o}(t)$ approach each other. Figure 24 shows the flow of the wave along the lattice in time. The solutions for $a=0.8$ are representative of 


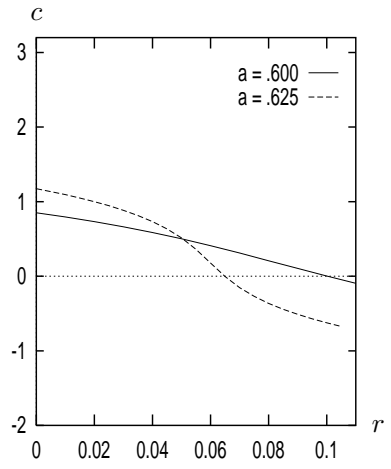

(i)

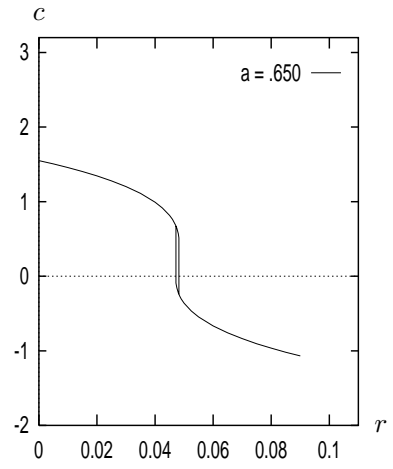

(ii)

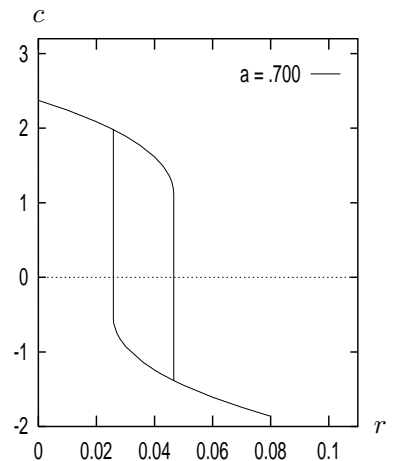

(iii)

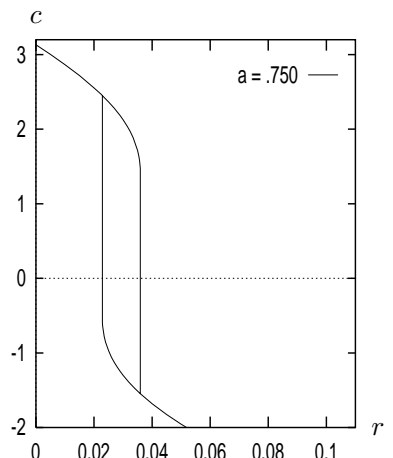

(iv)

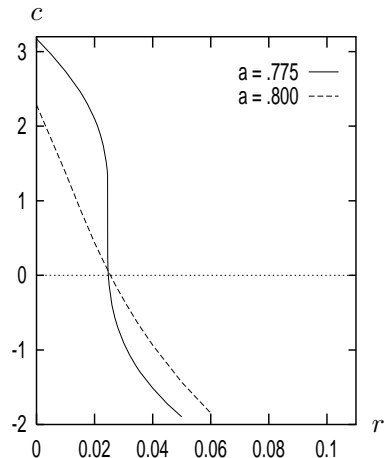

(v)

FIG. 19. Phase portrait: $c(t)=c_{e}(t)-c_{o}(t)$, the difference in wave speed, versus $r(t)=r_{e}-r_{o}=$ $\int_{0}^{t} c(s) d s$, the phase shift.

$r_{e}$

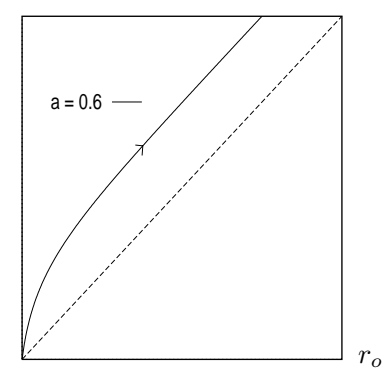

$r_{e}$

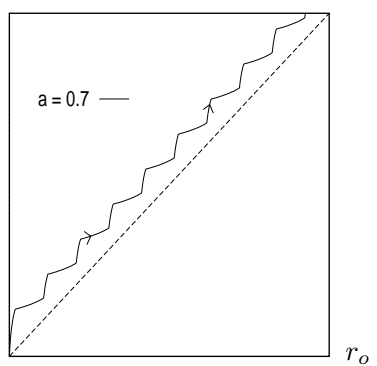

$r_{e}$

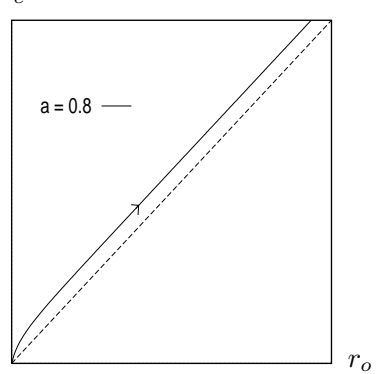

FIG. 20. Phase portraits of $r_{e}=\int_{0}^{t} c_{e}(s) d s$ versus $r_{o}=\int_{0}^{t} c_{o}(s) d s$ showing the flow with respect to time.

the solutions that both have single curvature changes in the solution profile.

Consider either the $a=0.6$ or the $a=0.8$ example, as the system proceeds in time, the initial phase conditions, solution profiles, and wave speeds are changing into a solution set that maps back into $u$ as a traveling wave moving through the lattice. Traveling wave solutions for both of these values of $a$ are stable, attracting solutions and phase conditions initially defined as if the lattice were uniformly spaced. The changing phase conditions imply that the variation in the diffusion coefficient $\alpha$ over 


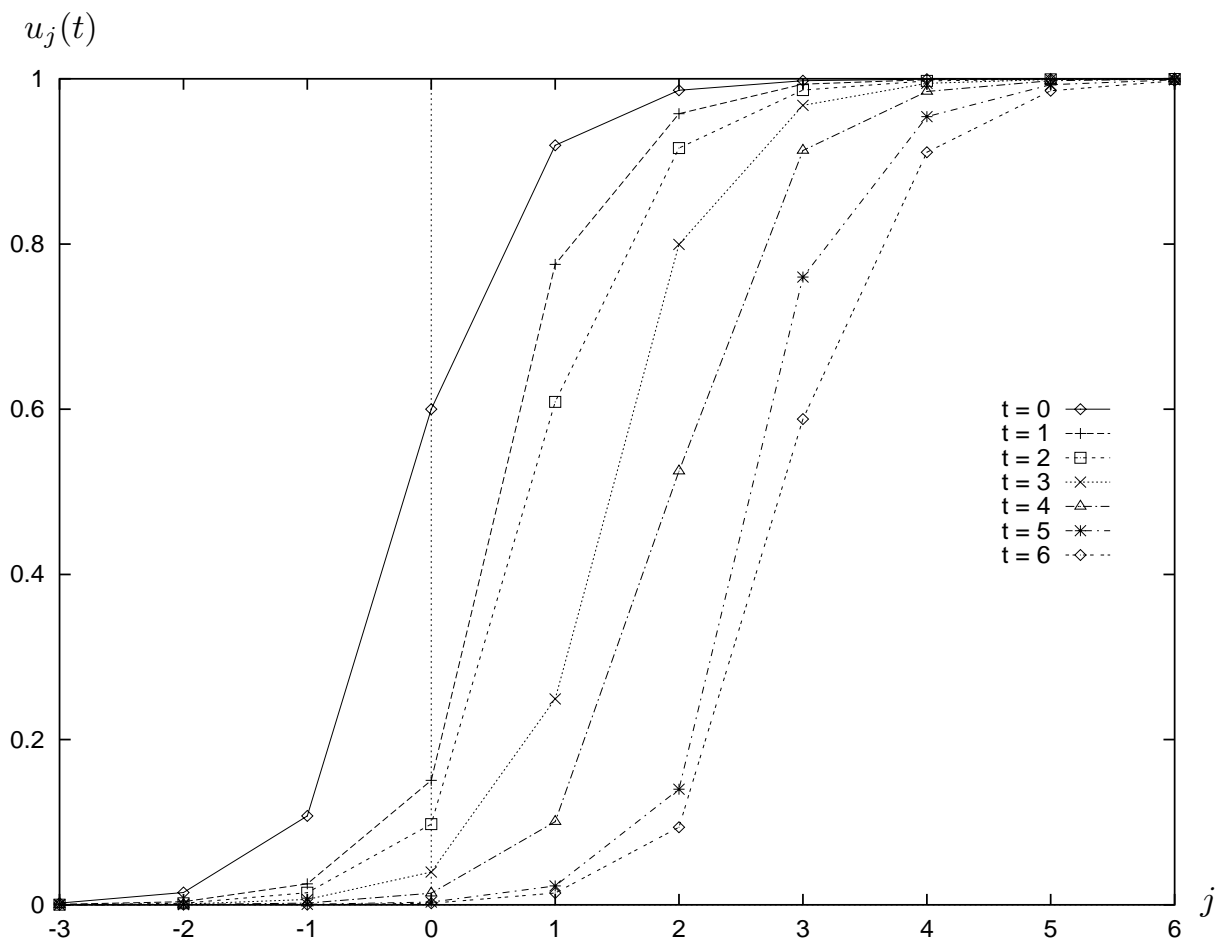

FIG. 21. The $u_{j}$ versus $j$ plot for $a=0.6$.

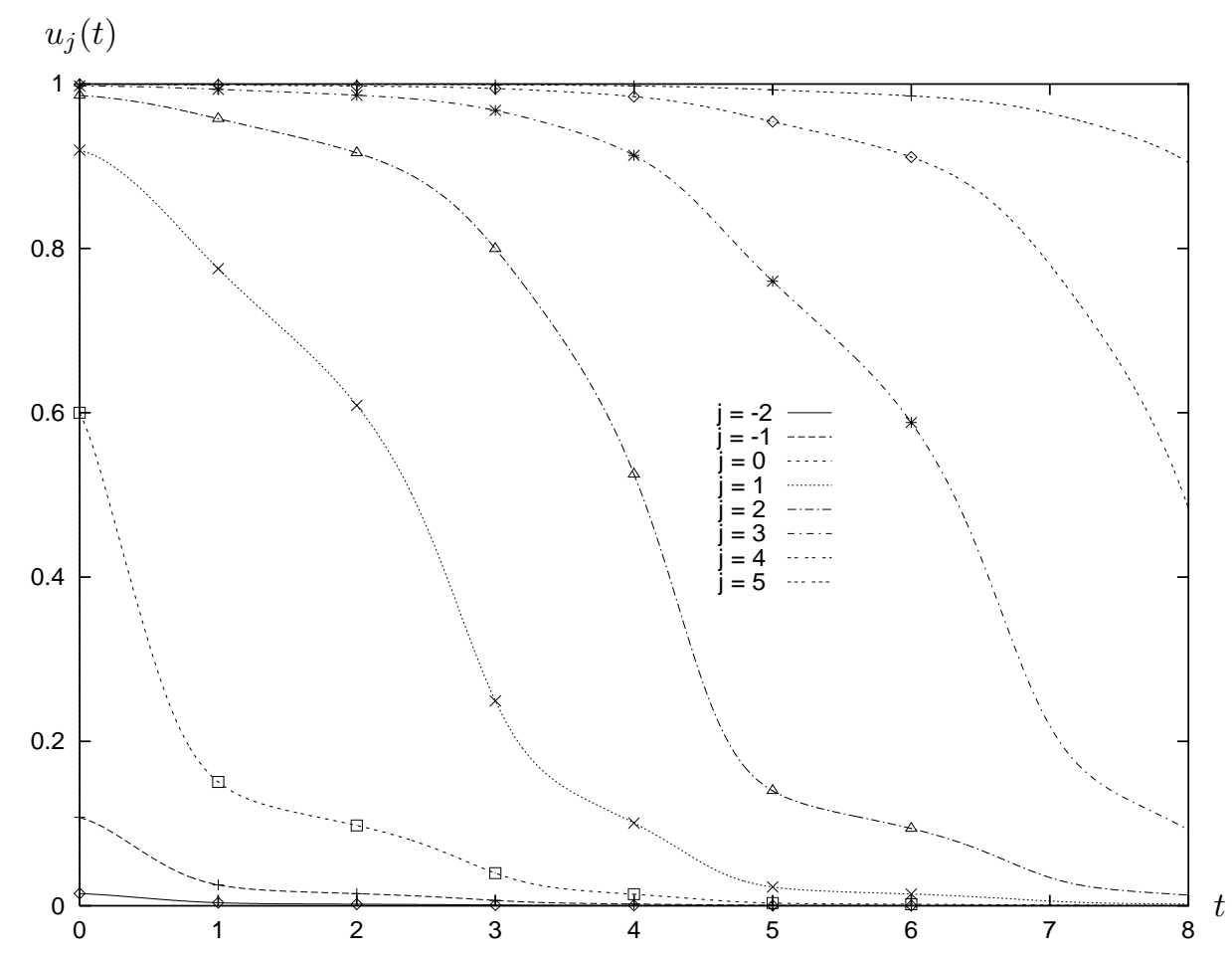

FIG. 22. The $u_{j}$ versus $t$ plot for $a=0.6$. 


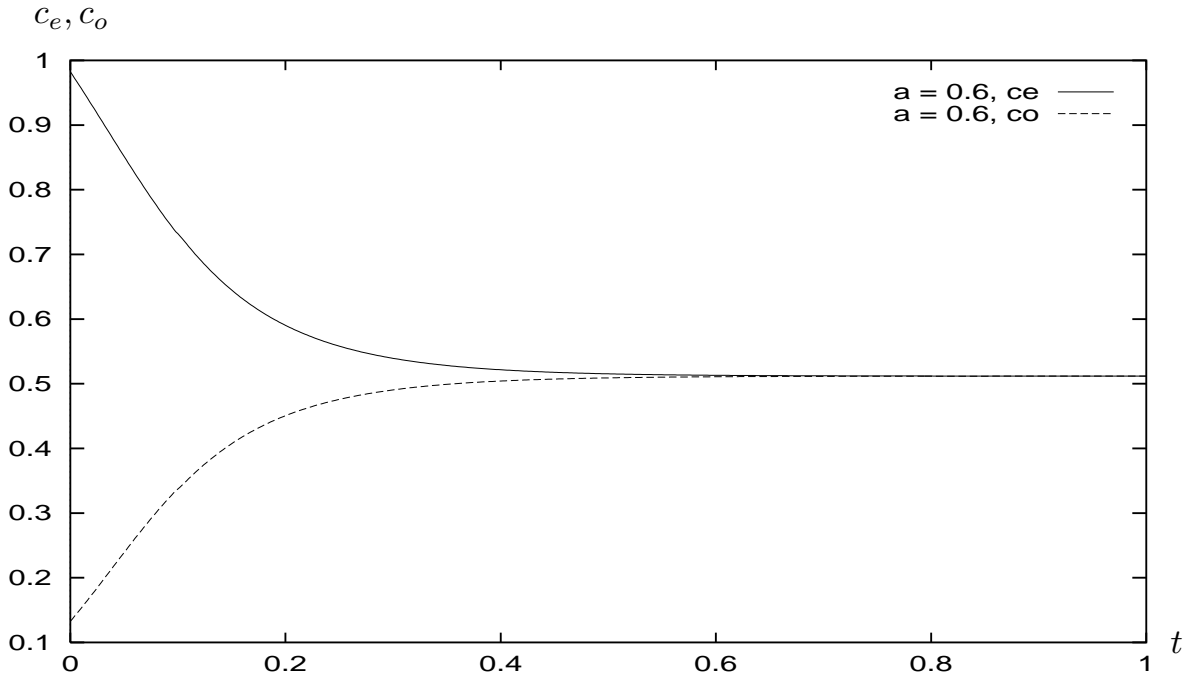

FIG. 23. The $c_{e}(t), c_{o}(t)$ plot for $a=0.6$.

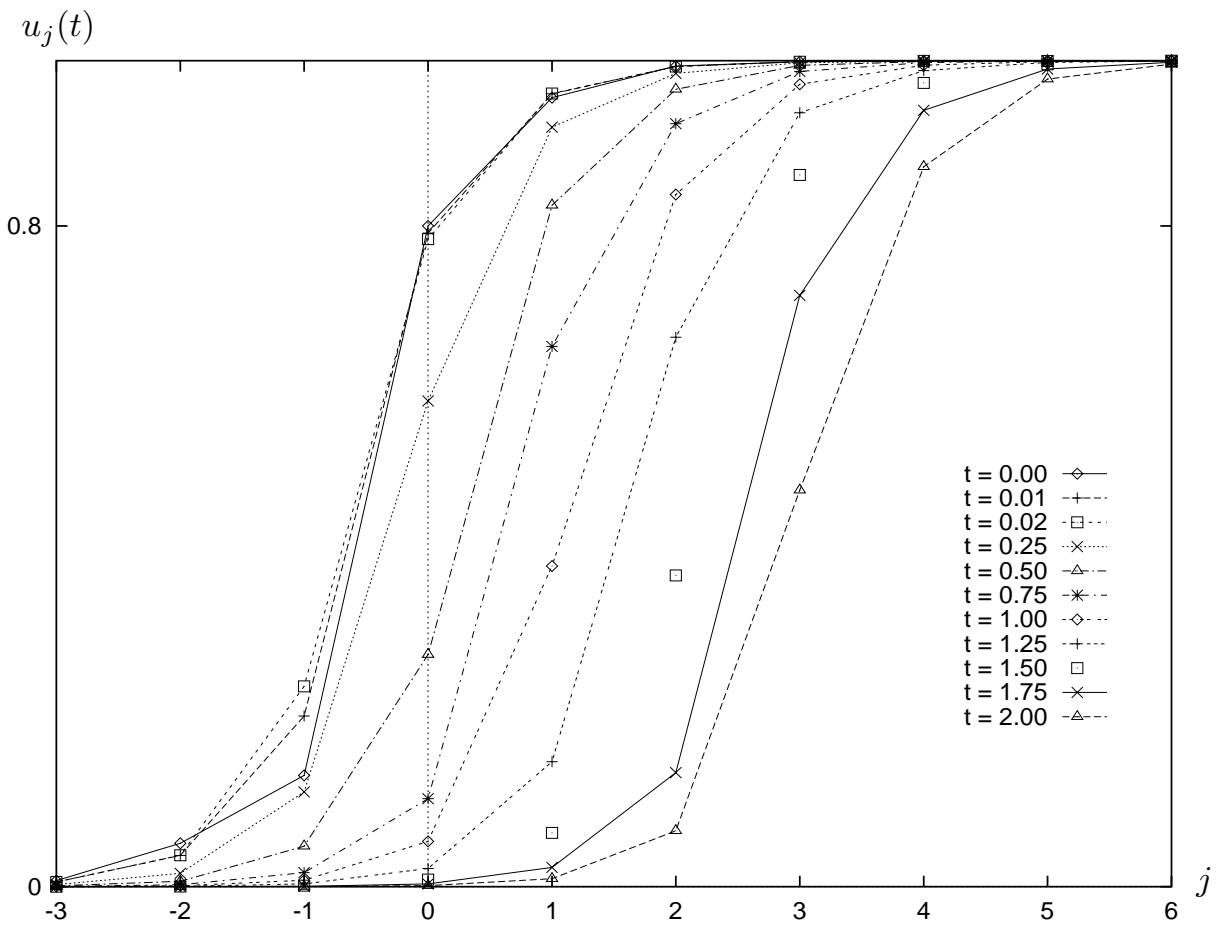

FIG. 24. The $u_{j}$ versus $j$ plot for $a=0.8$.

the lattice represents a variation in the lattice spacing. This seems to be an inherent property of our ansatz and the subsequent definition of the phase conditions. If the phase conditions were not allowed to change in time and remained uniformly spaced, then, because each of the two solution profiles in a pair would have different wave speeds, the solutions we have found do not represent traveling wave solutions in $u$. 


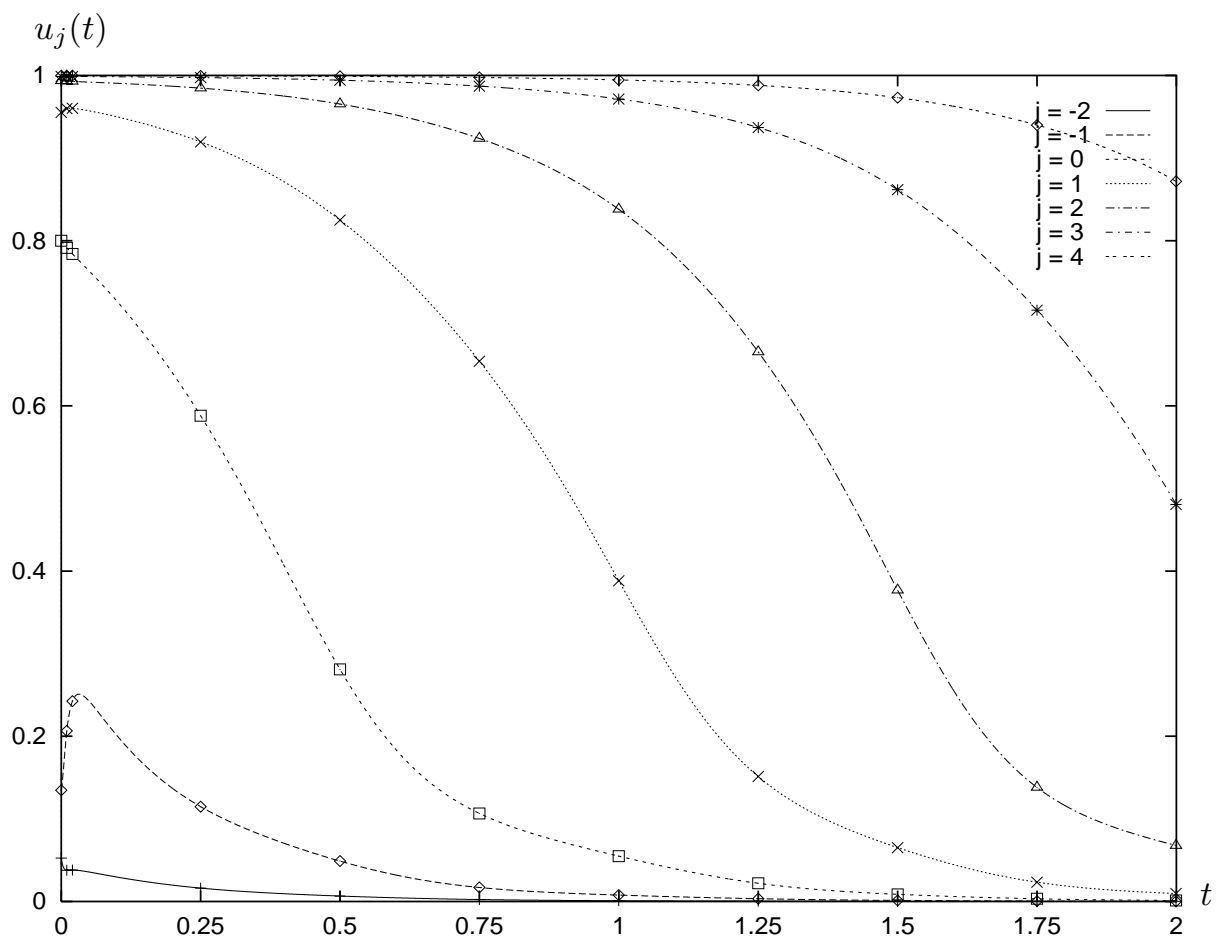

FIG. 25. The $u_{j}$ versus $t$ plot for $a=0.8$.

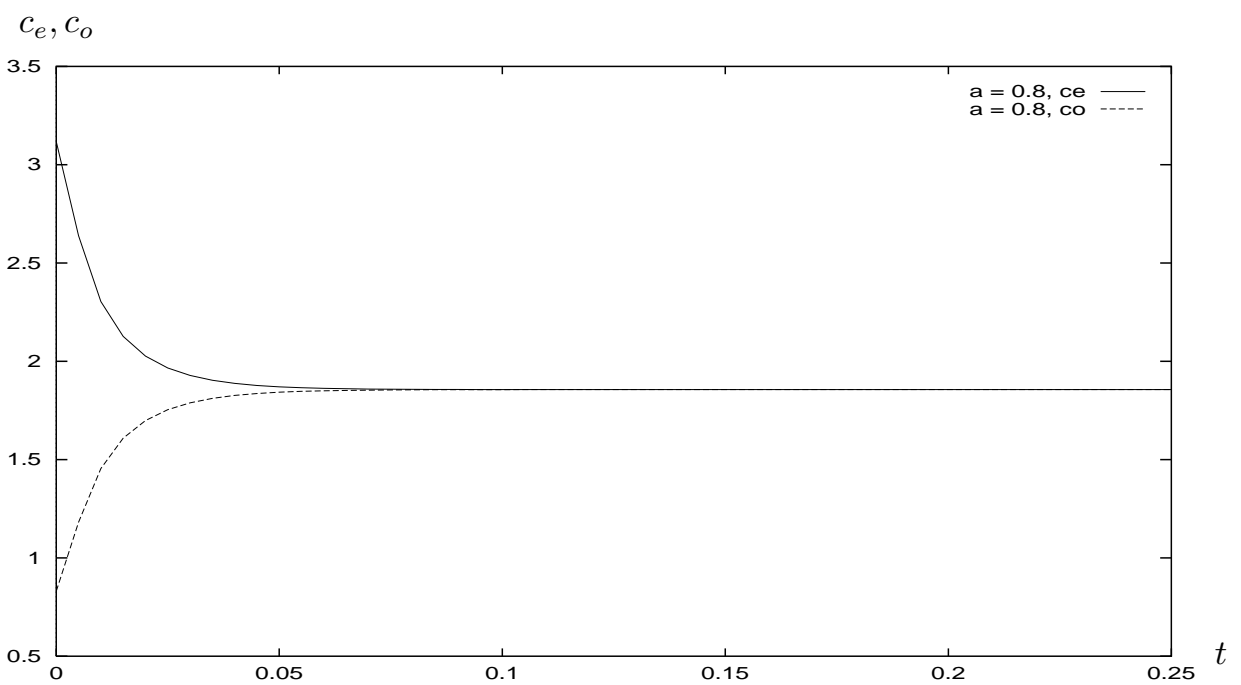

FiG. 26. The $c_{e}(t), c_{o}(t)$ plot for $a=0.8$.

8. Conclusions. In this paper we study traveling wave solutions to spatially discrete bistable reaction-diffusion equations with spatially periodic variable diffusion. We provide an analytical solution using the Fourier transform for the case when the bistable nonlinearity is represented by the piecewise linear function $f_{1}(1.5)$. The solution for general periodic diffusion is implicit although we provide an explicit solu- 
tion for diffusion of period two. This was accomplished by treating variable diffusion differential-difference equations as a mixed system of constant coefficient ordinary differential and differential-difference equations and fixing time. The period two diffusion coefficient case is provided to demonstrate the details of the solution method, in particular, the inverse transform. Since these solutions are fixed in time we observe that the wave along the lattice is monotone at any time $t$. The discontinuity in the nonlinearity $f_{1}$ causes $\varphi^{\prime}$ to be discontinuous. The period two diffusion coefficient case also gives us a chance to investigate the $a(c)$ relation for a nonconstant wave speed $c$.

We solve (1.9) numerically using the iterative method of [9], which solves without the use of continuation, with the BVP solvers COLNEW and COLMOD for the case when the bistable nonlinearity is the cubic polynomial $f_{2}(1.6)$. The numerical techniques we use to find the traveling wave solutions and wave speeds solve without information about the analytical solutions. Numerical examples are presented for fixed time $t=0$ and to illustrate how the waves evolve in time.

For fixed time $t=0$ we initially considered the case when the diffusion coefficient varies in space with period two. A pair of $(a, c)$ curves is presented (Figure 1) in which the wave speeds for the even and odd lattice sites act in much different ways with one of the curves mimicking the curve of the constant diffusion reaction-diffusion PDE and the other mimicking the curve of a constant diffusion spatially discrete system of reaction-diffusion equations. In the second example we considered homogeneous diffusion with period two wave speed and discovered a period doubling bifurcation from which the solutions at even and odd lattice sites move with different wave speeds. These are traveling solutions which are formed from two different monotone components. The third example demonstrates numerical solutions for period four spatially varying diffusion. The bifurcation regions which appear in the numerical examples occur for values of $a$ where the solutions profiles are changing from solutions with multiple changes of curvature to profiles with only one. Looking at the homogeneous diffusion case and allowing for only one wave speed, we discover that the solution profile $\varphi$ has a weak dependence on the parameter $a$ in this bifurcation region, i.e., $\partial \varphi / \partial a \approx 0$ for all $\xi \in \mathbb{R}$. We feel that the bifurcation phenomena are created by a combination of the spatially discrete Laplacian and the spinodal effects of the nonlinearity $f_{2}$. In addition, for the period two diffusion problem, there seem to be limitations on the possible values of the diffusion coefficients, such as the magnitude of their difference.

Study of the time evolution of period two spatially varying diffusion, section 7 , includes a study of the time dependence of the wave speed and of the phase shifts between the lattice points and demonstrates bifurcation points that are dependent on the parameter $a$. What is observed are two types of solutions, those in which the even and odd lattice sites evolve asymptotically to the same wave speed and those in which for all time there is an alternating pulsing behavior between the even and odd lattice sites (see the hysteresis loops in Figure 19). Using the $(a, c)$ curves for fixed time (e.g., Figure 14) for initial conditions for the $c_{j}(t)$, our numerical experiments, in the case of period two, suggest that for a range of the detuning parameter $a$ the even and odd lattice site wave speeds vary periodically while in a complementary range of $a$ the even and odd lattice site wave speeds approach a single wave speed as $t \rightarrow \infty$.

Variations in the diffusion coefficient of the materials model (1.7) and the biological model (1.8) may be small compared to what we allowed for in this work. The phase shift relations that appear in the traveling wave examples have an intuitive 
interpretation in terms of distance $\beta_{j}$ between lattice planes in (1.7), and the distance $L_{j}$ between nodes and the node length $\mu_{j}$ in (1.8). In terms of the model of a wave traveling through a material lattice, multiple changes in curvature profiles are solutions which exhibit effects of the underlying lattice. The single change on the curvature solution profile no longer shows the lattice effects.

\section{REFERENCES}

[1] U. M. Ascher, J. Christiansen, And R. D. Russell, Collocation software for boundary value ODEs, ACM Trans. Math. Software, 7 (1981), pp. 209-222.

[2] G. BADER And U. Ascher, A new basis implementation for a mixed order boundary value ODE solver, SIAM J. Sci. Statist. Comput., 8 (1987), pp. 483-500.

[3] W. J. BEYN, The numerical computation of connecting orbits in dynamical systems, IMA J. Numer. Anal., 9 (1990), pp. 379-405.

[4] J. W. CAHN, Theory of crystal growth and interface motion in crystalline materials, Acta Met., 8 (1960), pp. 554-562.

[5] J. W. Cahn, J. Mallet-Paret, and E. S. Van Vleck, Traveling wave solutions for systems of ODE's on a two-dimensional spatial lattice, SIAM J. Appl. Math., 59 (1999), pp. 455-493.

[6] J. R. CASH, G. Moore, AND R. W. Wright, An automatic continuation strategy for the solution of singularly perturbed linear two-point boundary value problems, J. Comput. Phys., 122 (1995), pp. 266-279.

[7] E. J. Doedel and M. J. Friedman, Numerical computation of heteroclinic orbits, J. Comput. Appl. Math., 25 (1989).

[8] C. E. Elmer and E. S. VAn Vleck, Analysis and computation of traveling wave solutions of bistable differential-difference equations, Nonlinearity, 12 (1999), pp. 771-798.

[9] C. E. Elmer and E. S. Van Vleck, A variant of Newton's method for the computation of traveling wave solutions of bistable differential-difference equations, 1999, submitted.

[10] G. FATH, Propagation failure of traveling waves in a discrete bistable medium, Phys. D, 116 (1998), pp. 176-190.

[11] P. C. FIfE, Diffusive waves in inhomogeneous media, Proc. Edinburgh Math. Soc. (2), 32 (1989), pp. 291-315.

[12] P. C. Fife AND L. HsiaO, The generation and propagation of internal layers, Nonlinear Anal., 12 (1988), pp. 19-41.

[13] M. J. Friedman And E. J. Doedel, Numerical computation and continuation of invariant manifolds connecting fixed points, SIAM J. Numer. Anal., 28 (1991), pp. 789-808.

[14] W.-Z. GAO, Threshold behavior and propagation for a differential-difference system, SIAM J. Math. Anal., 24 (1993), pp. 89-115.

[15] D. HANKERSON AND B. Zinner, Wavefronts for a cooperative tridiagonal system of differential equations, J. Dynam. Differential Equations, 5 (1993), pp. 359-373.

[16] J. P. KeEner, Propagation and its failure in coupled systems of discrete excitable cells, SIAM J. Appl. Math., 47 (1987), pp. 556-572.

[17] J. P. KEENER, The effects of discrete gap junction coupling on propagation in myocardium, J. Theoret. Bio., 148 (1991), pp. 49-82.

[18] J. Keener and J. Sneed, Mathematical Physiology, Springer-Verlag, New York, 1998.

[19] J. Mallet-PAREt, The Fredholm alternative for functional differential equations of mixed type, J. Dynam. Differential Equations, 11 (1999), pp. 1-48.

[20] J. MALlET-PARET, The global structure of traveling waves in spatially discrete dynamical systems, J. Dynam. Differential Equations, 11 (1999), pp. 49-128.

[21] J. Ortega and W. Rheinboldt, Iterative Solution of Nonlinear Equations in Several Variables, Academic Press, San Diego, CA, 1970.

[22] W. Shen, Traveling waves in time almost periodic structures governed by bistable nonlinearities I. Stability and uniqueness, J. Differential Equations, 159 (1999), pp. 1-54.

[23] W. Shen, Traveling waves in time almost periodic structures governed by bistable nonlinearities II. Existence, J. Differential Equations, 159 (1999), pp. 55-101.

[24] H. F. WeinBerger, Long-time behavior of a class of biological models, SIAM J. Math. Anal., 13 (1982), pp. 353-396.

[25] B. ZInNer, Stability of traveling wavefronts for the discrete Nagumo equation, SIAM J. Math. Anal., 22 (1991), pp. 1016-1020. 
[26] B. Zinner, Existence of traveling wavefront solutions for the discrete Nagumo equation, J. Differential Equations, 96 (1992), pp. 1-27.

[27] B. ZinneR, Wavefronts for a cooperative tridiagonal system of differential equations, J. Dynam. Differential Equations, 5 (1993), pp. 359-373.

[28] B. Zinner, G. HARris, And W. Hudson, Traveling wavefronts for the discrete Fisher's equation, J. Differential Equations, 105 (1993), pp. 46-62. 